\title{
Physical Processes and Chemical Reactions in Cometary Comae
}

\author{
S. D. Rodgers and S. B. Charnley \\ NASA Ames Research Center \\ W. F. Huebner and D. C. Boice \\ Southwest Research Institute
}

\begin{abstract}
A variety of physical and chemical processes are important in the comae of active comets near the Sun. We review the principal physical processes occurring in the outflowing gas and dust and their effects on thermodynamics of the coma. We describe the coupling between the physics and the chemistry and emphasize that any accurate model of the coma must include both. Chemically, there are a number of mechanisms capable of altering the initial chemical composition of the gas escaping from the nucleus surface. We assess the importance of these chemical processes, and discuss several recent models that follow the chemical evolution of the coma gas. The ultimate aim of most coma studies is to understand the nature of the icy nucleus, and we briefly review the major obstacles in using coma observations to infer the nucleus properties.
\end{abstract}

\section{INTRODUCTION}

Our knowledge of the composition and structure of comets has come primarily from studies of their comae. Astronomical observations of the coma can be made directly because small dust grains in the coma scatter and reflect light much more efficiently than the dark nucleus, and because the outgassing molecules emit distinct spectral lines at specific frequencies. Although several space missions have been sent to investigate comets, they have only probed the nucleus indirectly via photographic imaging and lowresolution spectroscopy. Not until the Rosetta mission sends a lander to the nucleus of a comet will we be able to directly access the material that resides in the nucleus. In contrast, there exists over a century of data pertaining to observations of cometary comae. It is also interesting to study the coma for its own sake - it provides an environment impossible to duplicate on Earth, and can be used as a laboratory to test theories of gas and plasma dynamics and photochemistry. Finally, the interaction of the coma with the solar wind can provide information on the properties of the solar wind and the interplanetary medium; in fact, the existence of the solar wind was first discovered via its interaction with cometary comae (Biermann, 1951).

\subsection{Structure of the Coma}

The coma is the cloud of dust and gas that surrounds the cometary nucleus. A schematic view of the coma structure is shown in Fig. 1. The size of the coma depends on how one defines it; for the purposes of this chapter, where we are discussing physical and chemical processes, we are concerned chiefly with the collisional coma, i.e., the inner region where particle collisions affect thermodynamics and chemistry of the gas. A rough estimate of the size of the collisional coma can be obtained by finding the cometocentric distance, $r$, at which the particle mean free path, $\Lambda$, equals r. For a Halley-type comet at $1 \mathrm{AU}$ from the Sun, this distance is typically several thousand kilometers for neutral-neutral collisions, and up to an order of magnitude larger for ion-molecule collisions, due to the enhanced cross-sections for ion-neutral interactions. The size of the collisional region is proportional to the total gas production rate, and so will increase as the comet approaches the Sun.

One can also loosely define a "molecular coma" within which most molecules survive against photodissociation. Strictly speaking, the size of this region will vary for different molecules, since all species have different photodissociation lifetimes. For water (and many other species as well), the lifetime in the solar radiation field at $1 \mathrm{AU}$ is $\approx 10^{5} \mathrm{~s}$, and so for a typical outflow velocity, $\mathrm{v}$, of $1 \mathrm{~km} \mathrm{~s}^{-1}$ the molecular coma will be $\sim 10^{5} \mathrm{~km}$ in size. For species such as ammonia and formaldehyde that are destroyed more rapidly, the coma will be an order of magnitude smaller, whereas more-stable species such as $\mathrm{CO}$ and $\mathrm{CO}_{2}$ will survive out to $10^{6} \mathrm{~km}$ or further. Photodissociation rates are proportional to the strength of the radiation field, and so molecular comae will shrink as the comet nears the Sun.

It is important to stress that the coma gas is not gravitationally bound to the nucleus, and so the coma is a transient phenomenon. Because sublimation from the nucleus surface is constantly replenishing the outflowing gas, the coma can often appear stable and unchanging. However, sudden changes in coma brightness and structure are common, with 


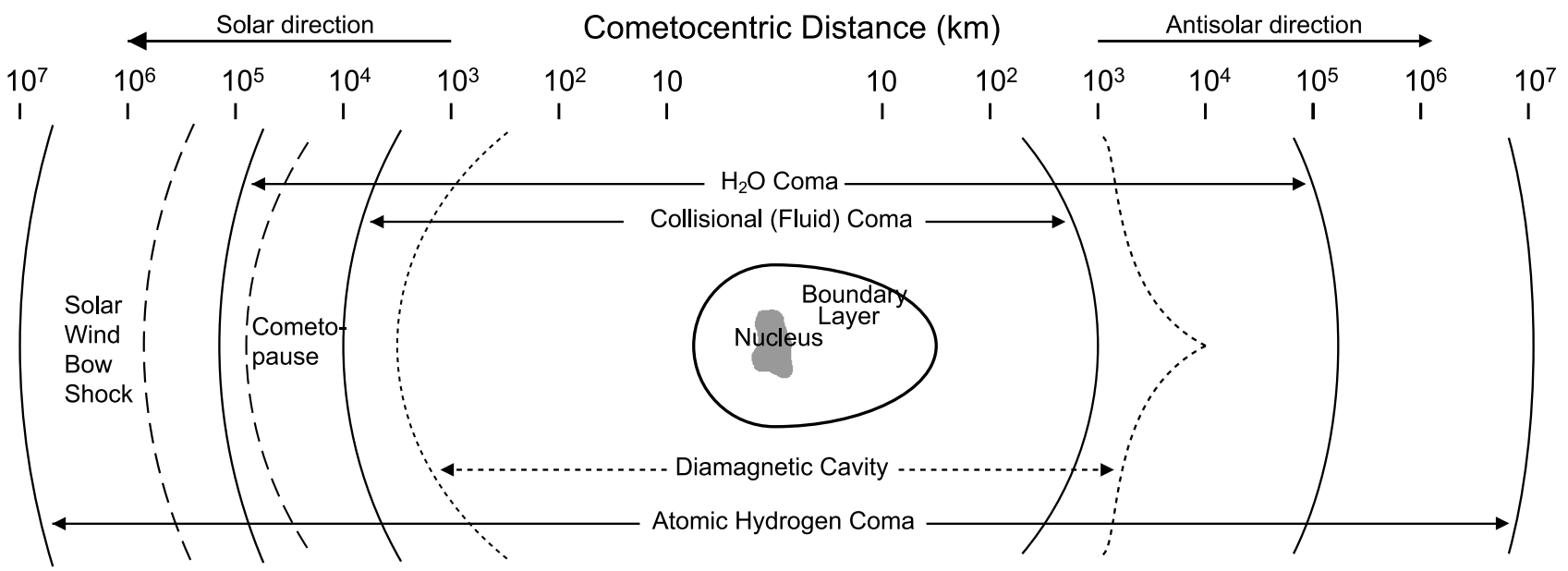

Fig. 1. Schematic illustration of the coma structure and its major physical regions for a moderate production rate at about 1 AU. Note the logarithmic distance scale.

features changing rapidly over timescales of several hours. Spatial structures are also seen in many comae, often in both the gas and dust components (although the structure in both may not be the same). Further discussions of these phenomena appear in Schleicher and Farnham (2004) and Combi et al. (2004).

The interaction of the solar wind with the coma results in complex structures in the plasma and magnetic fields (see Neubauer, 1991; Cravens, 1991; Ip, 2004; Lisse et al., 2004). A bow shock develops in the solar wind on the sunward side of the comet; within this region the solar wind "picks up" slow-moving coma species via charge exchange reactions. Eventually, as the wind encounters the denser gas in the inner coma it is decelerated, and at the cometopause it is diverted laterally around the inner coma. The interplanetary magnetic field also wraps around the comet, which results in a magnetic-field-free cavity surrounding the nucleus. The existence of this cavity in Comet Halley was demonstrated by Neubauer et al. (1986).

\subsection{Physical and Chemical Processes in the Coma}

Most of the processes that occur in the coma are initiated by the solar radiation field. Photons at ultraviolet (UV) wavelengths photodissociate and ionize the original parent molecules, producing "second-generation" reactive radicals, ions, and electrons. These ions and radicals can subsequently react with other species to form "third-generation" species. Examples include many of the protonated ions detected by the ion mass spectrometer on the Giotto probe in the coma of Comet Halley (Geiss et al., 1991). These processes are highly exoergic, and so can lead to species in excited states not normally populated at the low temperatures in the coma $(\mathrm{T} \approx 10-200 \mathrm{~K})$, as can direct absorption of solar photons (fluorescent pumping). Figure 2 illustrates the most important processes occurring in the coma.
The densities in the inner coma are sufficiently large that it is reasonable to consider the gaseous coma as a fluid, or more accurately as a mixture of several fluids (neutrals, ions, electrons, as well as different populations of dust grains, and suprathermal photodissociation products; although these latter components are not strictly fluids they can typically be described in terms of hydrodynamic variables, i.e., velocity and temperature). Hence, thermodynamic properties of the coma can be calculated from integration of the standard equations of fluid flow, assuming that the initial conditions at the nucleus surface are known (or can be estimated with some degree of accuracy). As the density decreases and $\Lambda$ increases, the fluid description becomes less applicable, and a transition to free molecular flow occurs. In this region, processes that affect a particular molecule cannot be assumed to affect the gas as a whole, and the properties of the outflowing gas are "frozen in" from the earlier collisional regime [see Crifo (1991) for a thorough discussion].

The coma gas also interacts with the entrained dust, and this affects both the dynamics and chemistry of the coma. For example, gas-dust drag in the very inner coma decelerates the outflowing gas to subsonic speeds (Marconi and Mendis, 1983). Chemically, dust grains may account for the "extended sources" of some coma molecules, where observations require that these molecules are injected into the coma, rather than released directly from the nucleus (e.g., Festou, 1999). This may result either from delayed sublimation of low-volatility material from the hotter grains, or from the actual breakup of the grains themselves. From measurements of the elementary dust composition in Comet Halley it is known that a significant fraction of refractory particles consist of organic (CHON) matter (Kissel et al., 1986). A variety of complex organics have been proposed to account for this material (Huebner and Boice, 1997). In particular, Huebner (1987) proposed that polyoxymethylene (POM, the $-\mathrm{CH}_{2} \mathrm{O}-$ polymer) could account for the ex- 


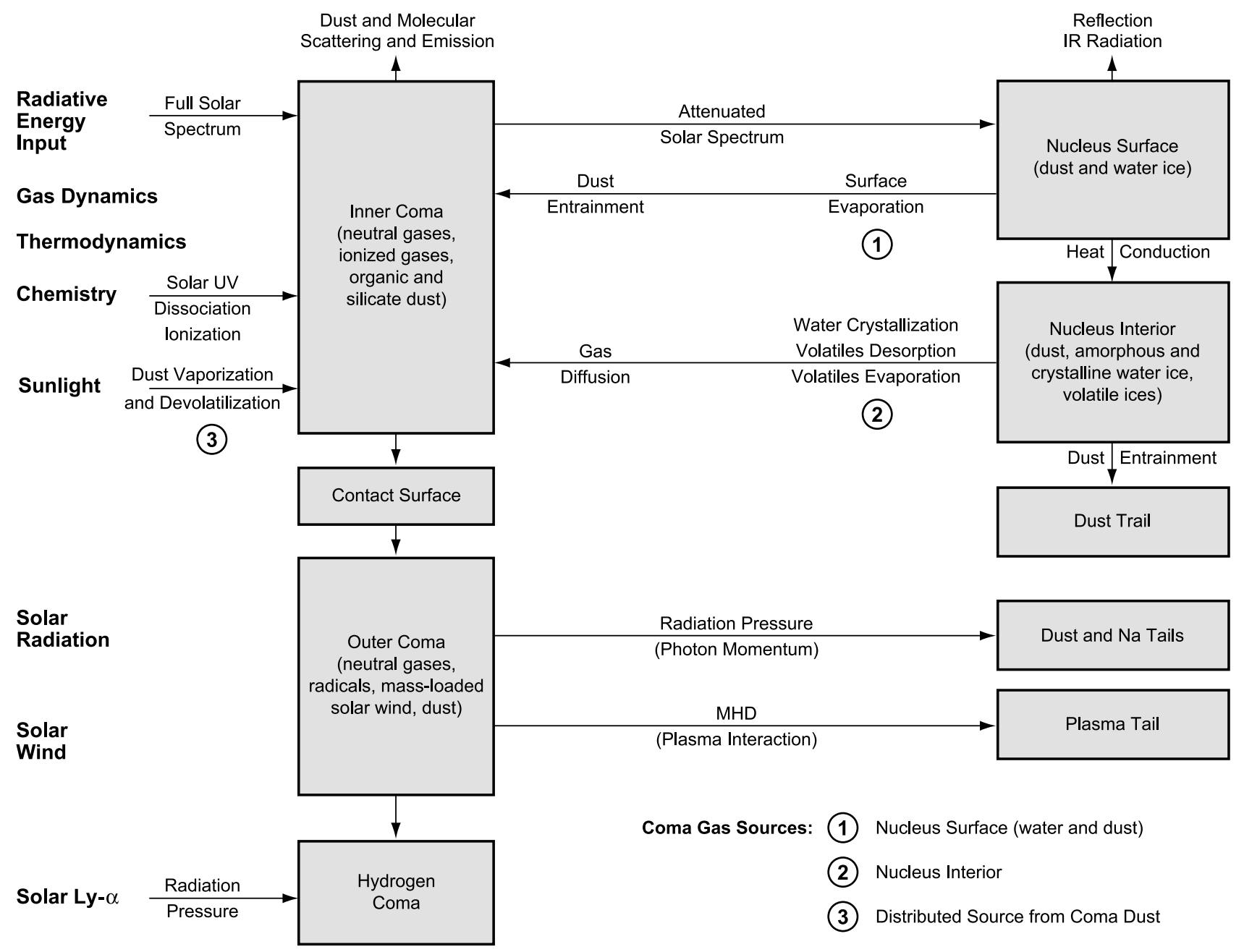

Fig. 2. The major radiative, physical, chemical, and thermodynamic processes comets, their coupling, and their interaction between the various cometary components: nucleus, coma gas, plasma, and dust (Huebner and Benkhoff, 1999).

tended source of formaldehyde in Comet Halley. Other macromolecules proposed to be potentially important in the coma include hexamethylenetetramine [HMT, $\mathrm{C}_{6} \mathrm{H}_{12} \mathrm{~N}_{4}$ (Bernstein et al., 1995)] and polyaminocyanomethylene [PACM, a - C $(\mathrm{CN})\left(\mathrm{NH}_{2}\right)$ - polymer (Rettig et al., 1992)].

\subsection{Brief History of Coma Modeling}

Prior to the middle of the twentieth century, many spectroscopic observations of cometary comae revealed the presence of molecular radicals such as $\mathrm{C}_{2}, \mathrm{C}_{3}, \mathrm{CH}, \mathrm{CN}, \mathrm{NH}$, $\mathrm{NH}_{2}, \mathrm{OH}$, and a few ions, including $\mathrm{CH}^{+}, \mathrm{CO}^{+}, \mathrm{CO}_{2}^{+}$, and $\mathrm{OH}^{+}$(e.g., Swings, 1943). In spite of its high abundance, $\mathrm{H}_{2} \mathrm{O}$ had not been detected but it was postulated to be present together with $\mathrm{CH}_{4}, \mathrm{CO}_{2}, \mathrm{NH}_{3}$, and $\mathrm{C}_{2} \mathrm{~N}_{2}$, consistent with the list of observed radicals and ions. Subsequently, Whipple $(1950,1951)$ postulated the icy conglomerate model for the comet nucleus based on observations of Comet Encke.
The first simple analytical model of molecular distributions in the coma was published by Haser (1957), who considered only photodissociation of parent molecules to form daughter and granddaughter molecules. However, as the importance of chemical reactions began to be appreciated in other astrophysical contexts, so it became clear that the high-density conditions in the inner coma $\left(\mathrm{n} \sim 10^{13} \mathrm{~cm}^{-3}\right.$ at the nucleus surface) meant that they were also likely to be important in comets. Such considerations, together with the advent of more powerful computers, led to the development of detailed chemical models (e.g., Oppenheimer, 1975; Giguere and Huebner, 1978; Huebner and Giguere, 1980; Mitchell et al., 1981; Biermann et al., 1982).

These early models had many simplifying assumptions, such as constant temperature and velocity profiles. The models confirmed that ion-molecule reactions were important in the inner coma, particularly proton transfer reactions followed by dissociative recombination. These models also 

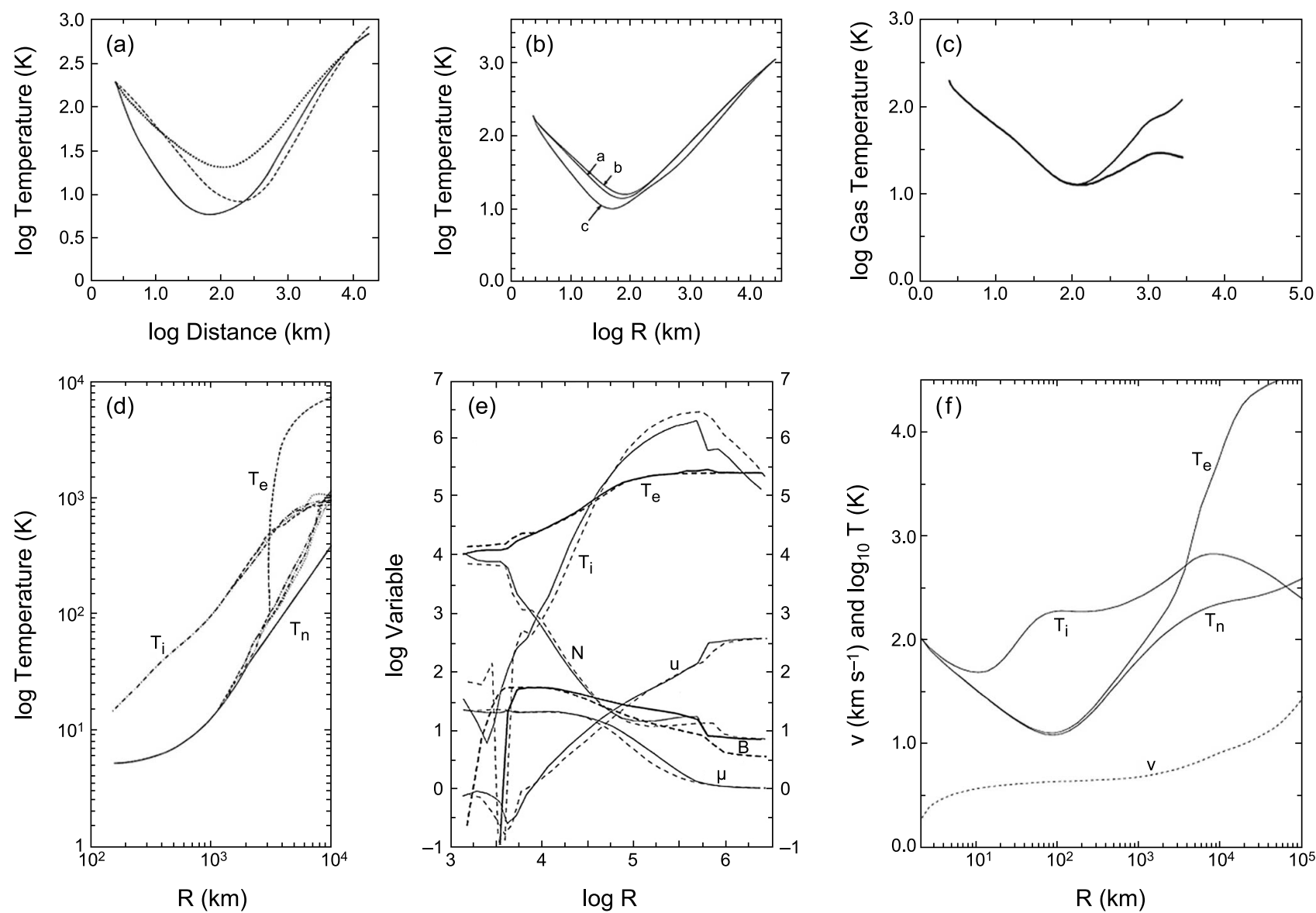

Fig. 3. Comparison of coma temperature profiles predicted by a variety of models. The upper panels show models for which a single fluid is considered, whereas the bottom panels show results for multifluid models that also include the effects of coma chemistry. In (a) and (b) the different lines correspond to different assumptions regarding the heating and cooling rates; (c) shows the difference between the results of hydrodynamic calculations (upper line) compared with Monte Carlo simulations (lower line). In the lower panels $T_{n}, T_{i}$, and $T_{e}$ refer to the temperatures of the neutral, ion, and electron fluids respectively. The different $T_{e}$ profiles in (d) refer to different assumptions regarding heat transfer from "hot" photoelectrons. In (e), u, N, $\mu$, and B are the velocity (km s $\left.{ }^{-1}\right)$, number density $\left(\mathrm{cm}^{-3}\right)$, mean molecular weight (amu), and magnetic field strength (nT), respectively. References: (a) Marconi and Mendis (1982); (b) Crovisier (1984); (c) Combi and Smyth (1988); (d) Körösmezey et al. (1987); (e) Schmidt et al. (1988); (f) Rodgers and Charnley (2002).

showed that the inner region of the coma is opaque to the solar UV field, and so accurate chemical models must include optical depth calculations. Later models included additional reactions such as radiative association, electron impact ionization and dissociation, and photodissociative ionization (e.g., Schmidt et al., 1988). Models have also been developed to account for the extended sources of $\mathrm{CO}$ and $\mathrm{H}_{2} \mathrm{CO}$ following the breakup of POM in the coma (Boice et al., 1990; Cottin et al., 2001).

Subsequent hydrodynamical models of the coma demonstrated that the assumptions of constant velocity and temperature were highly unrealistic (e.g., Marconi and Mendis, 1982, 1983, 1986; Gombosi et al., 1985, 1986; Combi and Smyth, 1988). In particular, adiabatic cooling of the gas as it initially expands away from the nucleus can lead to the temperature in the inner coma dropping to around $10 \mathrm{~K}$. Further out, as photodissociation and photoionization reactions become important, the temperatures rise, particularly that of the electrons. It was thus realized that the physics and chemistry are intimately coupled. For example, proton transfer reactions are the principal heat source for ions in the inner coma, and the rate of electron recombination reactions is greatly reduced in the outer coma when the electrons become extremely hot. This led to the development of combined hydrodynamic-chemical models (Huebner, 1985; Körösmezey et al., 1987; Wegmann et al., 1987; Rodgers and Charnley, 2002). The agreement between the various models is rather good; Fig. 3 presents the temperature profiles calculated by a number of different models. In the remainder of this chapter we shall concentrate on 
coma chemistry in the hydrodynamic picture, for the MHD and Monte Carlo treatments the reader is directed to Ip (2004) and Combi et al. (2004).

\section{OBSERVING THE COMA}

\subsection{Spectroscopy}

The great majority of cometary data is gathered via spectroscopy of the coma, and comes from all accessible regions of the electromagnetic spectrum. Molecular observations of coma composition are discussed in detail by Crovisier (2004), Feldman et al. (2004), and Bockelée-Morvan et al. (2004). For the purposes of this chapter we note that observations of spectral lines give a single globally averaged column density for the number of molecules in a particular energy level; interpreting this number requires a model of the coma. Specifically, one needs to know both n(r) and $\mathrm{f}_{\varepsilon}(\mathrm{r})$, where $\mathrm{n}$ is the number density and $\mathrm{f}_{\varepsilon}$ is the fraction of molecules in energy level $\varepsilon$ at cometocentric distance $r$. This distribution must then be convolved with the telescope beam profile for the appropriate Earth-comet distance. For parent species, it is usually sufficient to assume a Haser distribution for the density, i.e., $n \propto r^{-2} \exp \left\{-r / r_{\text {phot }}\right\}$, where $r_{\text {phot }}$ is the photodissociation scale-length at the appropriate heliocentric distance. For species formed in the coma the problem is more complicated; photodaughters in the outer coma can be modeled using the vectorial model of Festou (1981), or from the results of Monte Carlo calculations (see Combi et al., 2004). Molecules formed via coma chemistry require detailed chemical models to compute their radial density profiles.

The factor $f_{\varepsilon}$ is much more difficult to calculate, since it depends on a number of excitation and deexcitation mechanisms that operate simultaneously. In the inner coma collisions will dominate, and $\mathrm{f}_{\varepsilon}$ will approach the local thermodynamic equilibrium (LTE) value. Further out, collisions remain important, but may be too slow to depopulate photodaughters produced in highly excited states. As the electron temperature decouples from the neutrals, the collisional excitation will be higher for those species with large dipole moments that collide more rapidly with electrons (Xie and Mumma, 1992; Biver et al., 1999). Eventually, as all collisions become unimportant, the excitation will be determined by the balance between radiative pumping and fluorescent decay (Bockelée-Morvan and Crovisier, 1987). For lines that are optically thick, an extra layer of complication is added, in that a full radiative transfer model of the coma must be employed. If several lines of the same molecule from different energy levels can be observed, an estimate of the globally averaged coma temperature can be obtained (Biver et al., 1999). At radio wavelengths, the spectroscopic resolution is sufficiently narrow that expansion velocities can be obtained from line profiles (Bockelée-Morvan et al., 1990). Again, this value is a global average over the whole of the coma.

\subsection{In Situ Measurement}

Several space missions were sent to rendezvous with Comet Halley, and mass spectrometry of the coma was performed by the Giotto and Vega 1 and 2 probes (e.g., Krankowsky et al., 1986). Most recently, in situ analysis of a second comet occurred during the Deep Space 1 flyby of Comet Borrelly (e.g., Nordholt et al., 2003). Such measurements differ from spectroscopic data in that they give information that is local and instantaneous. This has the advantage of allowing the structure of the coma to be probed on small scales. For example, the extended sources of $\mathrm{CO}$ and $\mathrm{H}_{2} \mathrm{CO}$ in Comet Halley were discovered by mass spectrometry (Eberhardt et al., 1987; Meier et al., 1993). However, because the data only yield a single "snapshot" of the state of the coma, one potential pitfall in interpreting these measurements is that it is difficult to decide whether any anomalies represent previously undiscovered permanent coma features, or if they are due to transient phenomena.

In situ measurements give radial profiles of molecular and ionic abundances and gas properties such as density and temperature. Thus, these measurements represent the most stringent tests of chemical and dynamical models. For example, the importance of proton transfer reactions in the inner coma was proved by the results of the Giotto ion mass spectrometer (Geiss et al., 1991). The global properties of the coma as a whole are best obtained from spectroscopic measurements. In order to obtain the most understanding of the coma, a combination of in situ and global measurements are required, together with detailed modeling.

\section{COMA-NUCLEUS BOUNDARY}

A key region is in the vicinity of the nuclear surface, where the initial chemical and dynamical conditions of the outflowing coma are determined. In practice, given the extreme difficulties in accurately calculating the physics and chemistry in this region, most models of the coma simply assume some set of initial conditions as parameters to be input into the model. By comparing the model output with observations it is hoped that, in addition to understanding the processes occurring in the coma, the initial conditions can also be constrained with some degree of accuracy. However, because of the complexity of the boundary region, it is naive to assume that the conditions in the very inner coma can be easily related to the properties of the nucleus.

For example, although the initial chemical composition of the gas is similar to the composition of the nucleus ice, for some volatile molecules an additional contribution from subsurface sublimation fronts may also be important. A thorough discussion of the chemical differentiation and stratification in the nucleus appears in Prialnik et al. (2004). The total surface sublimation rate is controlled by the equation of energy balance at the nucleus surface, together with a Clausius-Clapeyron-type equation of state of the surface 
ices. For typical cometary parameters, the gas (primarily water) production rate is on the order of $10^{17} \mathrm{mols} \mathrm{cm}^{-2} \mathrm{~s}^{-1}$ at $1 \mathrm{AU}$ (Whipple and Huebner, 1976).

The dynamics of the circumnuclear boundary region are also extremely complicated, since the nucleus is likely to be heterogeneous on small scales. Sophisticated time-dependent three-dimensional models are required in order to calculate the gas and dust flow in this region, and are discussed further in Crifo et al. (2004). These models predict many interesting features, such as lateral flows and standing shocks. The possible chemical effects of these features have not yet been investigated, so it is not known whether or not they may play a role in generating new species. In the remainder of this chapter we will discuss the chemistry that occurs beyond the boundary region, where the gas flow is relatively smooth and almost spherically symmetric. We mention the possible chemical processing in the boundary layer simply to stress, again, that inferring the properties of the nucleus from coma observations is not a simple task.

The thickness of the boundary layer depends on the gas production rate. At large heliocentric distances the rate of gas production is small and the thickness of the boundary layer can be very large. In the extreme case, when the mean free path for collisions between gas molecules approaches infinity, a Maxwellian velocity distribution will never be established and the gas remains in free molecular flow. For a comet at $1 \mathrm{AU}$ with a gas production rate of $\approx 10^{17} \mathrm{~cm}^{-2} \mathrm{~s}^{-1}$ and $\mathrm{T} \approx 100 \mathrm{~K}, \Lambda$ is on the order of $20 \mathrm{~cm}$. Thus, the boundary layer is on the order of tens of meters on the subsolar side of the nucleus, and will be much larger on the nightside.

\section{DYNAMICAL-CHEMICAL MODELS OF THE COMA}

\subsection{Model Classification}

We begin with a brief review of the different types of models that have been used to model the coma. This is in no way meant to be an exhaustive list or rigid taxonomy of all models, but rather to give an idea of the strengths and weaknesses of different approaches, and what level of complexity is feasible and/or necessary.

The simplest hydrodynamical models of the coma are steady, spherically symmetric, single-fluid models that calculate radial profiles of $\mathrm{v}$ and $\mathrm{T}$. Such models were first used to investigate the principal heating and cooling mechanisms operating in the coma, and showed that $\mathrm{T}$ can vary significantly throughout the coma (e.g., Marconi and Mendis, 1982; Crovisier, 1984; Huebner, 1985) (see Fig. 3). Such models also serve as the foundation for more complex calculations, such as models that are multidimensional (Kitamura, 1986), time-dependent (Gombosi et al., 1985), or include several fluids (Marconi and Mendis, 1986). In the case of multidimensional or time-dependent models, the computational demands can be very large, because these calculations involve simultaneously solving large sets of coupled partial differential equations (PDEs), as opposed to a relatively small number of ordinary differential equa- tions (ODEs) for one-dimensional, steady models. Models that considered separate neutral, ion, and electron fluids found that chemical reactions in the inner coma were an important heat source, especially for the ions (Körösmezey et al., 1987).

Chemically, the simplest models ignore the dynamics, and assume constant outflow velocities and temperatures (Giguere and Huebner, 1978). Other models take the gas physics into account in some basic fashion, for example, by assuming a sudden jump in the electron temperature at some arbitrarily chosen radius (Lovell et al., 1999). However, because chemical reaction rates depend on the gas temperature and density, accurately calculating the chemistry requires an accurate description of the coma dynamics. In addition, as discussed above, an accurate dynamical model should include the effect of chemical reactions. Therefore, the coma chemistry and physics should be solved simultaneously, and a multifluid, dynamical-chemical model is the minimum necessary to study coma chemistry. Such models were first developed by Körösmezey et al. (1987) (albeit with a limited chemistry) and Schmidt et al. (1988). In the following section we describe the basic components of such a model.

A separate category of coma models calculate the gas properties via Monte Carlo techniques (Combi and Smyth, 1988; Hodges, 1990). These models represent the most accurate descriptions of the coma, since they neglect the assumption, common to all hydrodynamic models, that the gas behaves as a fluid. However, these models are also extremely computationally demanding. Including a detailed chemistry in these models would be prohibitive. Given the fairly good agreement between Monte Carlo models and fluid-dynamic treatments in the inner collisional coma, it is not necessary to use such models when modeling the coma chemistry.

\subsection{Simple Multifluid Hydrodynamic Models}

Simple, spherically symmetric models, appropriate for a steady flow, have previously been employed to investigate the chemistry of the collisional inner coma (e.g., Huebner, 1985; Wegmann et al., 1987; Schmidt et al., 1988; Konno et al., 1993; Canaves et al., 2002), and we now describe the key ingredients of such a model; a more detailed description is given in Rodgers and Charnley (2002). In reality, as we have discussed, cometary comae are not symmetric, and rapid temporal variations are observed. Despite this, such models remain useful since (1) pressure differences in the very inner coma tend to even out rapidly, leading to fairly symmetric spherical outflows in the gas coma (Crifo, 1991); and (2) the time taken for a particular parcel of gas to reach a distance of $4 \times 10^{3} \mathrm{~km}-$ roughly corresponding to the edge of the collisional regime where bimolecular physical and chemical processes cease to significantly affect the gas - is around $1 \mathrm{~h}$. The most important deviations from this model occur for the ions and electrons at large cometocentric distances, where the interaction with the solar wind becomes important. In this case, multidimensional models 
are necessary (Schmidt et al., 1988). Nonetheless, because magnetic fields are excluded from the inner coma where most of the chemistry occurs, the plasma flow in these regions will trace the near-spherical neutral gas outflow. For a chemically reacting multicomponent flow we have to consider the interaction of the predominately neutral gas and the charged plasma of ions and electrons, and the relatively massive dust grains. We also need to treat the suprathermal photoproduced hydrogen atoms as an individual component (see section 4.3.3). Here we specifically describe the gasplasma coupling. Assuming a steady, spherically symmetric flow, and neglecting gravitational and magnetic fields, the hydrodynamic conservation equations for particle number, mass, momentum, and energy for the neutral gas are

$$
\begin{gathered}
\frac{1}{\mathrm{r}^{2}} \frac{\mathrm{d}}{\mathrm{dr}}\left(\mathrm{r}^{2} \mathrm{nv}\right)=\mathrm{N} \\
\frac{1}{\mathrm{r}^{2}} \frac{\mathrm{d}}{\mathrm{dr}}\left(\mathrm{r}^{2} \rho \mathrm{v}\right)=\mathrm{M} \\
\frac{1}{\mathrm{r}^{2}} \frac{\mathrm{d}}{\mathrm{dr}}\left(\mathrm{r}^{2} \rho \mathrm{v}^{2}\right)+\frac{\mathrm{dP}}{\mathrm{dr}}=\mathrm{F} \\
\frac{1}{\mathrm{r}^{2}} \frac{\mathrm{d}}{\mathrm{dr}}\left[\mathrm{r}^{2} \mathrm{v}\left(\frac{\rho v^{2}}{2}+\frac{\gamma}{\gamma-1} \mathrm{P}\right)\right]=\mathrm{G}+\mathrm{Fv}-\frac{1}{2} M v^{2}
\end{gathered}
$$

where $\mathrm{n}$ is the number density, $\rho$ is the mass density, $\mathrm{v}$ is the velocity, $\mathrm{P}$ is the pressure, and $\gamma$ is the ratio of specific heats. The source terms N, M, F, and G represent, respectively, the net generation rate per unit volume of particles, mass, momentum, and thermal energy (heat). It is assumed that the fluid behaves as an ideal gas. Equation (1) can be rearranged to give

$$
\frac{\mathrm{dn}_{\mathrm{s}}}{\mathrm{dr}}=\frac{\mathrm{N}_{\mathrm{s}}}{\mathrm{v}}-\frac{\mathrm{n}_{\mathrm{s}}}{\mathrm{v}} \frac{\mathrm{dv}}{\mathrm{dr}}-\frac{2 \mathrm{n}_{\mathrm{s}}}{\mathrm{r}}
$$

where the subscript s represents a particular chemical species. Similarly, the mass conservation equation becomes

$$
\frac{\mathrm{d} \rho}{\mathrm{dr}}=\frac{\mathrm{M}}{\mathrm{v}}-\frac{\rho}{\mathrm{v}} \frac{\mathrm{dv}}{\mathrm{dr}}-\frac{2 \rho}{\mathrm{r}}
$$

After some algebra, one can derive equations for the radial derivatives for the velocity and temperature of the neutral gas

$$
\begin{array}{r}
\frac{d v}{d r}=\frac{1}{\rho v^{2}-\gamma P}\left(F v-(\gamma-1) G-M v^{2}+\frac{2 \gamma P v}{r}\right) \\
\frac{d T}{d r}=\frac{2 T}{r}+\frac{1}{n k v}\left(F v-M v^{2}-N k T+\frac{d v}{d r}\left(n k T-\rho v^{2}\right)\right)
\end{array}
$$

Each of the three fluids in the coma (neutrals, ion, electrons) has a distinct temperature, and similar equations can be derived for $\mathrm{dT}_{\mathrm{i}} / \mathrm{dr}$ and $\mathrm{dT}_{\mathrm{e}} / \mathrm{dr}$, as well as for the mass density gradient of the plasma. For the plasma, a slight complication arises, since charge conservation and the strength of the Coulomb force ensures that $\mathrm{n}_{\mathrm{e}}=\mathrm{n}_{\mathrm{i}}$ and $\mathrm{v}_{\mathrm{e}}=\mathrm{v}_{\mathrm{i}}$ throughout the coma. Therefore, one must solve for the plasma velocity $\mathrm{dv}_{\mathrm{e}} / \mathrm{dr}$ by considering the total contributions from both ions and electrons. Once the hydrodynamic source terms have been defined, and the fluid properties at the comet surface prescribed, it is possible to numerically integrate the resulting system of differential equations to obtain the coma physical and chemical structure.

In practice, for steady flows such as these there can exist mathematical singularities where a fluid encounters a transonic point, since at these points the denominator in equation (7) becomes zero. When a dust component is included, such a $0 / 0$ singularity is encountered close to the nucleus, as the expanding neutral gas then has to undergo a subsonicsupersonic transition. Similarly, further out in the coma, where the electron temperature becomes very high, the plasma sound speed increases to the point that the plasma undergoes a supersonic-subsonic transition. For time-dependent flows, where a system of PDEs is solved, singularities do not occur (e.g., Körösmezey et al., 1987; Körösmezey and Gombosi, 1990). In the case of steady flows, special numerical techniques, or simplifying approximations, are needed to treat such 0/0 singularities (e.g., Marconi and Mendis, 1983, 1986; Gail and Sedlmayr, 1985). It should be emphasized that, although "simple" by the standards of recent coma dynamics models (e.g., Combi et al., 2004; Crifo et al., 2004), even steady flow models require a higher level of sophistication relative to other areas of astrochemical modeling (as in interstellar clouds or circumstellar envelopes, for example). Model temperature distributions obtained for a comet similar to Hyakutake at $1 \mathrm{AU}$ are shown in Fig. 3f.

\subsection{Heating and Cooling Mechanisms}

4.3.1. Elastic scattering. Elastic collisions transfer momentum and energy between the three fluids. For ionneutral collisions, if the scattering is assumed to be isotropic in the center-of-mass frame, the mean amount of thermal energy imparted to the neutrals per collisions can be calculated via

$$
\begin{gathered}
\hat{\mathrm{G}}_{\mathrm{n}}(\mathrm{i}-\mathrm{n}, \text { elastic })= \\
2 \mathrm{~m}_{\mathrm{n}} \mathrm{m}_{\mathrm{i}}\left(\mathrm{m}_{\mathrm{n}}+\mathrm{m}_{\mathrm{i}}\right)^{2}\left[\frac{3}{2} \mathrm{~K}\left(\mathrm{~T}_{\mathrm{i}}-\mathrm{T}_{\mathrm{n}}\right)+\mathrm{m}_{\mathrm{i}} \frac{\left(\mathrm{v}_{\mathrm{n}}-\mathrm{v}_{\mathrm{e}}\right)^{2}}{2}\right]
\end{gathered}
$$

where $m_{n}$ and $m_{i}$ are the masses of the particles involved. A similar expression holds for the ion fluid heat source term per collision. The total thermal energy source term due to elastic scattering is obtained by summing equation (9) over all collisions. This can be done either by assuming a generic value for the mean mass of each fluid, or by actively summing the contributions of the most frequent collisions, which will involve the ions $\mathrm{H}_{3} \mathrm{O}^{+}, \mathrm{CH}_{3} \mathrm{OH}_{2}^{+}$, and $\mathrm{NH}_{4}^{+}$collid- 


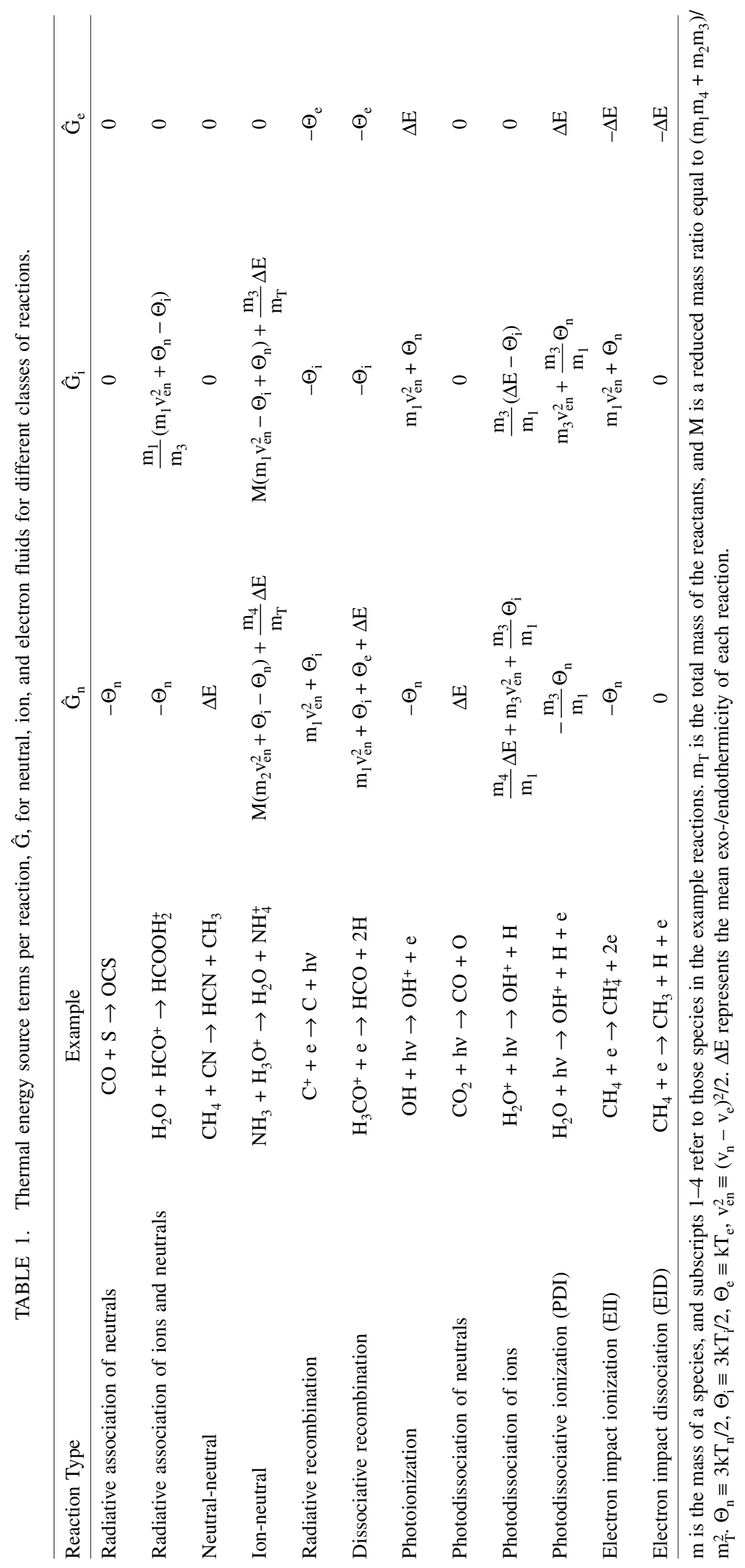


ing with $\mathrm{H}_{2} \mathrm{O}, \mathrm{CO}$, and $\mathrm{CO}_{2}$. In either case, the rate coefficient for the collisions must be known; a value equal to the Langevin value, $\sim 10^{-9} \mathrm{~cm}^{3} \mathrm{~s}^{-1}$, is typically assumed. Note that the source term in equation (9) can be considered as a simplified form of the general expression for reactive ionmolecule collisions (see line 4 in Table 1).

For electron-neutral elastic scattering, the most important collision partner is water. Collision rates for e- $\mathrm{H}_{2} \mathrm{O}$ mixtures were measured by Pack et al. (1962; see also Körösmezey et al., 1987), and the mean heat transfer per collision can be obtained from equation (9) with the additional assumption that the electron mass can be neglected. Also, because the cross section declines with electron temperature, one finds that the mean thermal energy of a colliding electron is $\mathrm{kT}_{\mathrm{e}}$, not $3 \mathrm{kT}_{\mathrm{e}} / 2$ (Draine, 1986). The rate of energy transfer between ions and electrons was calculated by Draine (1980).

4.3.2. Inelastic scattering. Inelastic collisions can lead to molecules in highly excited states. These will then decay with the emission of a photon, and if this photon is able to escape from the coma without being reabsorbed, the energy is lost. Inelastic collisions involving $\mathrm{H}_{2} \mathrm{O}$ are the most important; water molecules can be excited by collisions with other $\mathrm{H}_{2} \mathrm{O}$ molecules or with electrons. A semiempirical formula for the energy loss due to the former was derived by Shimizu (1976). However, radiation trapping in the inner coma means that the effective cooling rate is much less (Crovisier, 1984). This can be roughly accounted for by introducing an optical depth factor (Schmidt et al., 1988). The energy removed by inelastic e- $\mathrm{H}_{2} \mathrm{O}$ collisions was calculated by Cravens and Körösmezey (1986). Again, accurate calculations must include the effects of radiation trapping in the inner coma.

4.3.3. Thermalization of energetic photoproducts. The majority of photolytic reactions result in the production of either atomic or molecular hydrogen, and due to their low masses these products also have the largest share of the excess energy of the reaction. Hence, the dominant heating mechanism in the coma is thermalization of fast $\mathrm{H}$ and $\mathrm{H}_{2}$ particles. It was realized by Ip (1983) that in the outer coma many of these particles will escape from the coma before thermalization, thus removing an important energy source for the neutral fluid. A variety of calculations of this effect have been performed (e.g., Huebner and Keady, 1984; Combi and Smyth, 1988). Although the results are qualitatively similar, the radius at which escape becomes important can vary by almost an order of magnitude in different models (Crifo, 1991).

The most important reaction forming fast $\mathrm{H}$ atoms (hereafter denoted $\mathrm{H}_{\mathrm{f}}$ ) is photodissociations of water

$$
\mathrm{H}_{2} \mathrm{O}+\mathrm{hv} \rightarrow \mathrm{OH}+\mathrm{H}_{\mathrm{f}}
$$

The mean excess energy of reaction (10) is $3.4 \mathrm{eV}$, but as pointed out by Crovisier (1989), a large fraction of this energy may go into ro-vibrational excitation of the $\mathrm{OH}$ radical. Laboratory experiments on water photodissociation have shown that $\mathrm{OH}$ radicals with rotational energy levels of $\mathrm{J}>$
50 can be formed (e.g., Harich et al., 2001). Therefore, the kinetic energy of the $\mathrm{H}_{\mathrm{f}}$ atom is likely to be in the range $1-2 \mathrm{eV}$, implying a velocity of $\approx 15-20 \mathrm{~km} \mathrm{~s}^{-1}$. This has been confirmed by observations of numerous comets (Festou et al., 1983; McCoy et al., 1992). In addition to being the principal heating mechanism in the inner coma, these suprathermal atoms may also drive high-energy chemical reactions in this region. This will be discussed in section 5.4.

4.3.4. Chemical reactions. The hydrodynamic source terms due to chemical reactions depends on the reaction type and the masses and temperatures of the species involved. In order to accurately calculate the effects of chemistry on thermodynamics it is necessary to compute the source term due to each individual reaction and then multiply this by the total reaction rate (i.e., reactions $\mathrm{cm}^{-3} \mathrm{~s}^{-1}$ ), and then sum the total for all reactions. Mass source terms are trivial to calculate; e.g., the ionization of water increases the ion fluid mass by $18 \mathrm{amu}$, and decreases the neutral fluid mass by the same amount. Source terms for momentum and energy transfer are not so simple, however. Draine (1986) derived the expressions appropriate for several of the most common reaction types occurring in a multifluid flow and this methodology can be extended to include all reaction types that occur in the coma (Rodgers and Charnley, 2002). The resulting heat source terms per reaction, $\hat{G}$, are listed in Table 1.

A comparison of the effectiveness of each of the heating and cooling mechanisms discussed in this section is shown in Fig. 4.

\subsection{Gas-Dust Coupling}

The drag force exerted by the gas on a dust particle depends on the relative drift velocity between gas and dust, $\mathrm{v}_{\text {drift }}=\mathrm{v}_{\mathrm{g}}-\mathrm{v}_{\mathrm{d}}$, and can be written

$$
\mathrm{F}_{\text {drag }}=\mathrm{n}_{\mathrm{d}} \sigma_{\mathrm{d}} \rho_{\mathrm{g}} \frac{\mathrm{C}_{\mathrm{D}}}{2} \mathrm{v}_{\text {drift }}^{2}
$$

where $\mathrm{n}_{\mathrm{d}}$ and $\sigma_{\mathrm{d}}$ are the number density and cross section of the dust grains. $C_{D}$ is the drag coefficient, which accounts for the sticking of the molecules on the dust particles, the viscosity of the gas, and the shape of the particle. It also depends on the density of the gas. The drag coefficient is usually expressed in terms of the Reynolds number, $\mathrm{Re}=$ $2 r_{d} \rho_{g} v_{\text {drift }} / \mu$, where $\mu$ is the viscosity of the gas. For low Reynolds numbers, the drag coefficient reduces to the Stokes value, $C_{D}=24 / R e$. A good fit to the drag coefficient over a wide range of Reynolds number was provided by Putnam (1961)

$$
\begin{aligned}
& C_{D}=\frac{24}{\operatorname{Re}}\left(1+\frac{\operatorname{Re}^{2 / 3}}{6}\right) \quad(\operatorname{Re}<1200) \\
& C_{D}=0 \quad(1200<\operatorname{Re}<1200)
\end{aligned}
$$

The above discussion applies to gases that can be treated as a continuous medium, i.e., $\Lambda$ is much smaller than the size of the dust particle. If this is not the case, Knudsen flow ap- 

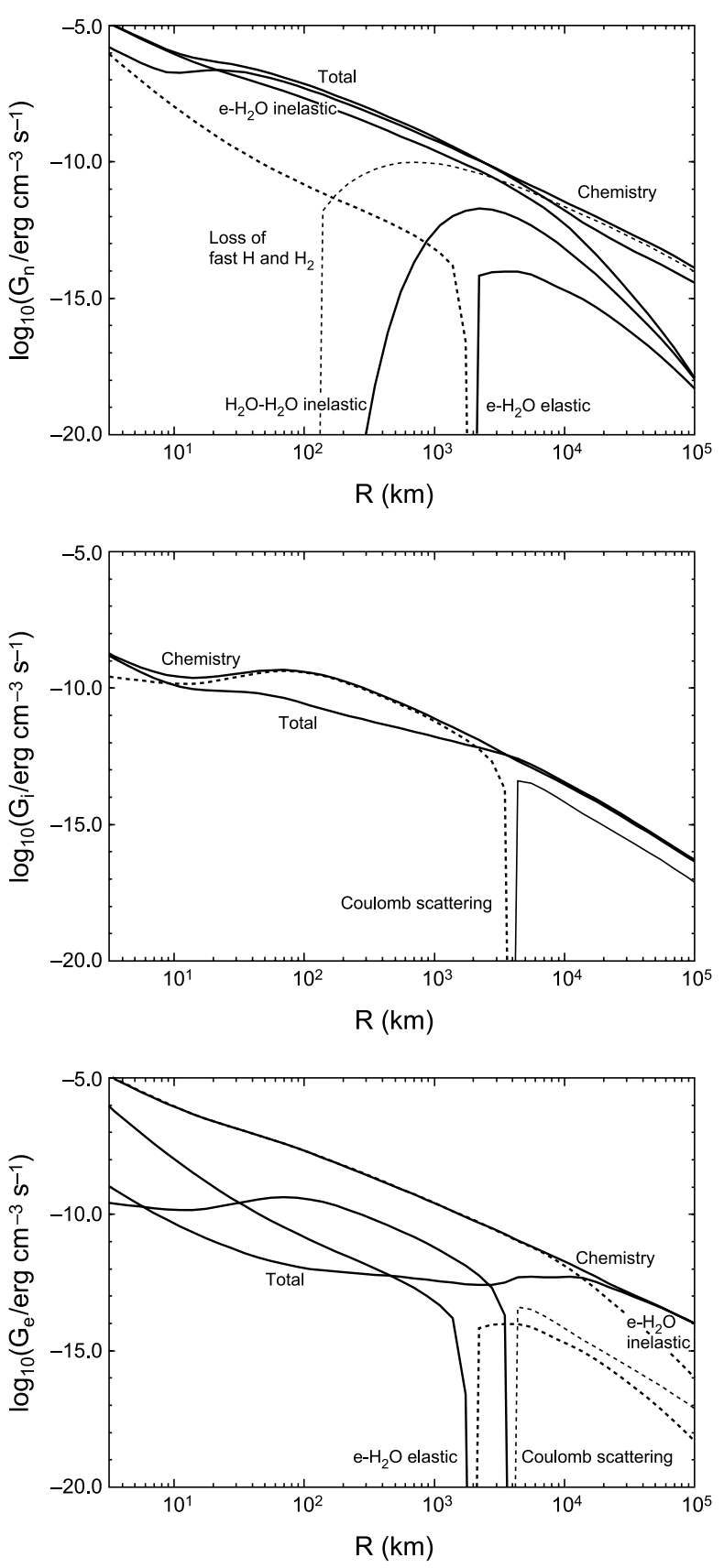

Fig. 4. Comparison of different heating and cooling mechanisms for the neutral (n), ion (i), and electron (e) fluids (from Rodgers and Charnley, 2002). For each process, the total heat source term, $\mathrm{G}\left(\mathrm{erg} \mathrm{cm}^{-3} \mathrm{~s}^{-1}\right)$, is shown; solid and dashed lines represent heating and cooling respectively. Processes considered include chemical reactions (this also includes photochemistry), loss of fast hydrogen atoms and molecules, elastic collisions between e-n, i-n, and i-e (Coulomb scattering) fluids, and inelastic water-water and electron-water collisions.

plies and a correction must be applied to the drag coefficient by dividing it by a factor $[1+\operatorname{Kn}(2.492+0.84 \exp \{-1.74 /$ $\mathrm{Kn}\})$ ] ( Friedlander, 1977), where Kn is the Knudsen number (the ratio of $\Lambda$ to the particle size). For a typical comet at $1 \mathrm{AU}, \Lambda \sim 20 \mathrm{~cm}$ (see section 3), and so Knudsen flow dominates for typical dust particles in cometary comae and dust tails. For larger dust particles, the deceleration due to gravity must also be taken into account. Equating the drag force given by equation (11) with the gravitational force gives the size of the largest particles that can be carried away by the sublimating gas.

Gas-dust collisions can also heat or cool the colliding gas particles. The temperature of dust grains is determined by the energy balance between solar heating, gas-drag heating, and cooling by reradiation. For a steady flow, the evolution of the dust temperature can be calculated from

$$
\mathrm{v}_{\mathrm{d}} \mathrm{C}_{\mathrm{d}} \frac{\mathrm{dT}_{\mathrm{d}}}{\mathrm{dr}}=\dot{\mathrm{E}}_{\mathrm{abs}}+\dot{\mathrm{E}}_{\mathrm{drag}}-\dot{\mathrm{E}}_{\mathrm{rad}}
$$

where $C_{d}$ is the heat capacity and the terms on the righthand side refer to the energy sources/sinks mentioned above. In general, these terms will depend on the shape, size, and mineralogy of the grains. For example, small grains are hotter than large grains, and, since they are more absorptive, Fe-bearing silicate grains tend to be hotter than $\mathrm{Mg}$ rich ones (Harker et al., 2002). The energy transfer between the gas and dust, $\dot{\mathrm{E}}_{\mathrm{drag}}$, also affects the gas flow. In general, this term is calculated from an expression of the form

$$
\dot{\mathrm{E}}_{\text {drag }}=\mathrm{n}_{\mathrm{g}} \sigma_{\mathrm{d}} \mathrm{C}_{\mathrm{H}}\left(\mathrm{T}_{\mathrm{g}}-\mathrm{T}_{\mathrm{d}}\right)
$$

where $\mathrm{C}_{\mathrm{H}}$ is the thermal accommodation coefficient. Because collisions between dust grains are rare, dust grains are coupled to the gas but not to each other. Therefore, an accurate description of the dusty coma requires the use of numerous dust components, each corresponding to a different population of grains with particular properties (e.g., size, shape, chemical composition). The acceleration of each component is obtained from equation (11) and the temperature from equation (14).

\section{COMA CHEMISTRY}

\subsection{Photochemistry}

The principal chemical processes occurring in the coma are photodissociation and ionization of the parent molecules. Accurate photodissociation rates are essential in order to model the coma and to interpret observational data. Huebner et al. (1992) compiled laboratory and theoretical data on a large number of important coma species; integrating over the solar spectrum yields photorates appropriate for both "quiet" and "active" solar photon fluxes. Despite the gargantuan nature of this undertaking, however, many gaps in our knowledge remain. Only a handful of species have been measured over a large range of wavelengths; many important radicals are unstable under laboratory conditions and so are extremely difficult to investigate. In many experiments, although the rates are well determined, the branching ratios among different sets of possible products 

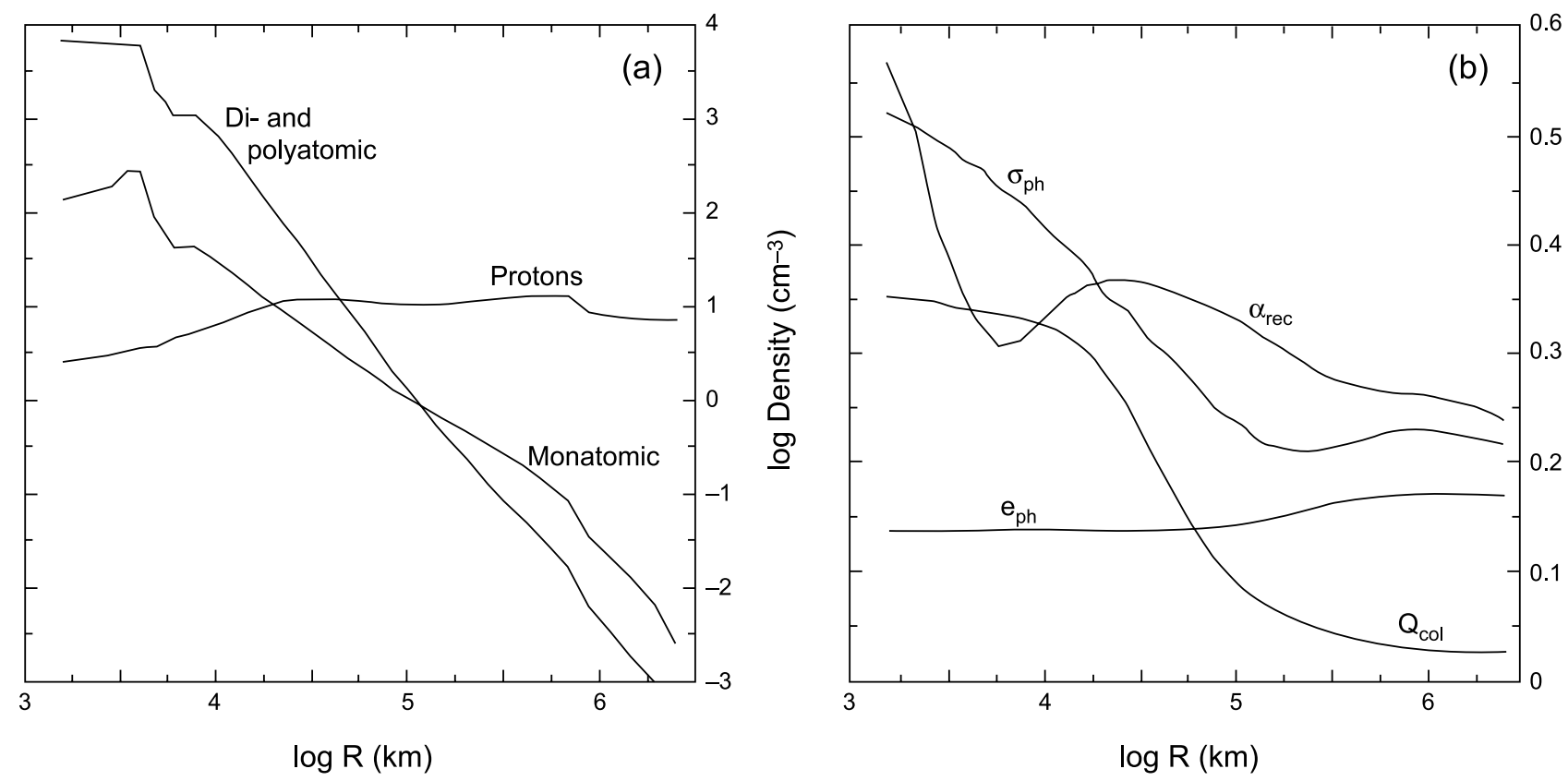

Fig. 5. Coma ion chemistry (Schmidt et al., 1988). (a) Relative densities of ionized species in the coma. (b) Cometocentric variations of important quantities in ion-molecule chemistry. Effective rate coefficients and average surplus energy for photoprocesses [ $\sigma_{\mathrm{ph}}$ $\left(10^{-6} \mathrm{~s}^{-1}\right)$ and $\mathrm{e}_{\mathrm{ph}}\left(10^{2} \mathrm{eV}\right)$ ]; effective rate coefficient for electron recombination and effective cross-section for ion-neutral elastic collisions $\left[\alpha_{\mathrm{rec}}\left(10^{-6} \mathrm{~cm}^{3} \mathrm{~s}^{-1}\right)\right.$ and $\left.\mathrm{Q}_{\mathrm{col}}\left(10^{-14} \mathrm{~cm}^{2}\right)\right]$.

are not well constrained. Thus, laboratory experiments are vital in order to further our understanding of coma chemistry (e.g., Jackson et al., 2002).

As the most important species in the coma, the photochemistry of water is of key importance. Due to its importance in atmospheric chemistry, water has been studied extensively in the lab. Details of all the possible product channels are discussed in Feldman et al. (2004). Here, we note a couple of important points. First, the dominant dissociation channel results in $\mathrm{OH}+\mathrm{H}$. Studies of $\mathrm{OH}$ are therefore an excellent proxy for determining the cometary water production rate (as long as the $\mathrm{OH}$ excitation mechanisms are fully understood). The $\mathrm{H}_{\mathrm{f}}$ atoms produced in this reaction are the principal source of heating in the inner coma, and may also drive a suprathermal chemistry. Second, the ionization of water occurs at a rate of $\approx 3 \times 10^{-7} \mathrm{~s}^{-1}$ at $1 \mathrm{AU}$. Hence, for an outflow velocity of $1 \mathrm{~km} \mathrm{~s}^{-1}$, the fractional ionization in the coma increases as $\mathrm{n}_{\mathrm{e}} / \mathrm{n}\left(\mathrm{H}_{2} \mathrm{O}\right) \sim(\mathrm{r} /$ $\mathrm{km}) \times 3 \times 10^{-7}$. This gives a rough estimate of the amount of material that can potentially be affected by ion-molecule reactions.

\subsection{Ion-Molecule Reactions}

For molecules with permanent dipole moments, the dipole-charge interaction results in an extra attractive force between the reactants. In this case the rate coefficient can be calculated from the average-dipole-orientation theory of Su and Bowers (1973). Eberhardt and Krankowsky (1995) showed that the resulting rate coefficients can be parameterized in the form

$$
\mathrm{k}\left(\mathrm{T}_{\mathrm{in}}\right)=\mathrm{k}(300)\left[1+\beta\left(\sqrt{\frac{300}{\mathrm{~T}_{\mathrm{in}}}}-1\right)\right]
$$

where $\beta$ depends on the dipole moment, $\mu$, polarizability, $\alpha$, and a "locking constant." $T_{\text {in }}$ is a mass-weighted mean kinetic temperature of the reactants. $\beta$ can be calculated from the expression $\beta=\mu /(\mu+\alpha)$, with $\mu$ measured in Debye and $\alpha$ in $\AA^{3}$ (Rodgers and Charnley, 2002).

In many ways the chemistry in the coma is analogous to the chemistry that occurs in interstellar hot cores. A "proton cascade" transfers protons from species with low proton affinity, i.e., $\mathrm{OH}\left(\mathrm{H}_{2} \mathrm{O}^{+}\right)$, to species with larger proton affinities, i.e., $\mathrm{NH}_{3}\left(\mathrm{NH}_{4}^{+}\right)$. Detailed chemical models are necessary to calculate how the ionization is apportioned among different ions, which is essential when interpreting in situ ion mass spectrometry data (Geiss et al., 1991). Many interstellar chemical schemes are lacking in a number of important proton transfer reactions, most notably from methanol to ammonia (Rodgers and Charnley, 2001a). The inclusion of a full set of such reactions is a prerequisite for an accurate calculation of ionic abundances. Figure 5 shows the degree of ionization and the abundances of different families of ions in the coma, together with various parameters affecting the plasma chemistry. 
As discussed earlier, the chemistry and hydrodynamics of the coma are intimately coupled. For example, proton transfer reactions are exothermic by typically a few electron volts $(\mathrm{eV})$. Therefore, the ions formed via these reactions will have significant energies, and this effect keeps the ions in the inner coma at a warmer temperature than the neutral fluid (Körösmezey et al., 1987). Also, the rates of many chemical reactions are temperature dependent. This is most important for dissociative recombination reactions of ions and electrons; when the value of $\mathrm{T}_{\mathrm{e}}$ reaches extremely large values in the outer coma, such reactions effectively switch off, resulting in a larger ionization fraction (see Lovell et al., 1999; Bockelée-Morvan et al., 2004, Fig. 1).

Despite the importance of ion-molecule reactions in determining ionic abundances, it turns out that these reactions are not an efficient source of new, stable neutral molecules. For example, Irvine et al. (1998) proposed that proton transfer to $\mathrm{HCN}$ followed by recombination could account for the HNC seen in Comet Hale-Bopp. However, models that include a comprehensive ion-molecule chemistry (i.e., involving $\mathrm{CH}_{3} \mathrm{OH}$ and $\mathrm{NH}_{3}$ ) show that the amount of $\mathrm{HNC}$ actually produced is almost 100 times less than observed (Rodgers and Charnley, 1998, 2001a). This is illustrated in Fig. 6. Similar calculations on the formation of large organics also show that these species are produced only in small abundances (Rodgers and Charnley, 2001b). A corollary of this is that one can show that the $\mathrm{HCOOH}, \mathrm{CH}_{3} \mathrm{OCHO}$, and $\mathrm{CH}_{3} \mathrm{CN}$ observed in Hale-Bopp (Bockelée-Morvan et al., 2000) cannot be formed by chemical reactions in the coma.

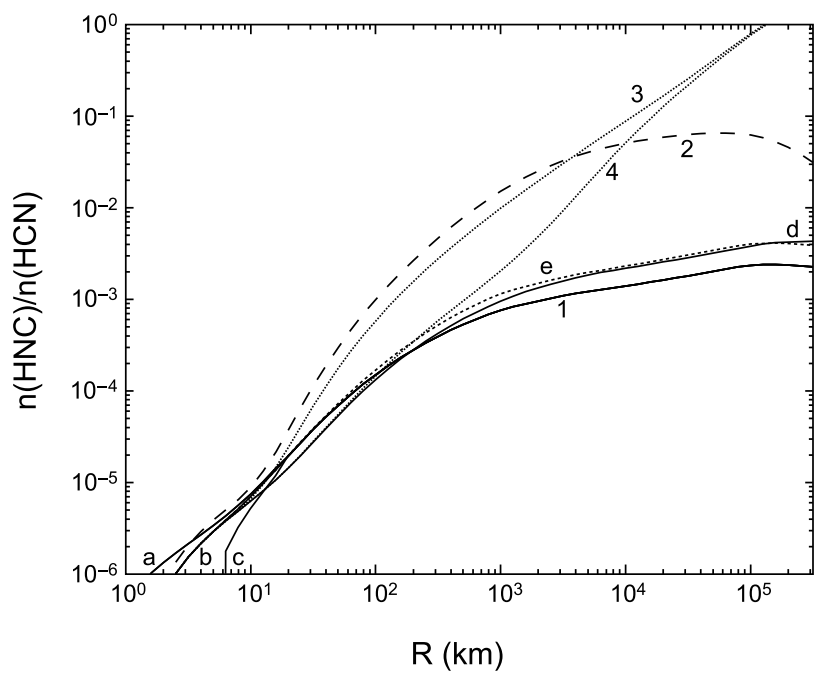

Fig. 6. $\mathrm{HNC} / \mathrm{HCN}$ ratios for a variety of $\mathrm{HNC}$ formation mechanisms (Rodgers and Charnley, 2001c). (1) Ion-molecule chemistry (curves a-e result from altering model parameters, such as the initial $\mathrm{HCN}$ abundance, recombination branching ratios, etc.). (2) Isomerization of $\mathrm{HCN}$ by $\mathrm{H}_{\mathrm{f}}$ atoms. (3) Photodestruction of an unknown parent. (4) As for (3), but assuming the parent of $\mathrm{HNC}$ also has an extended source.

\subsection{Neutral-Neutral Reactions}

An alternative source of new species in the coma may be provided by neutral-neutral reactions. Because photodissociation occurs around 100 times faster than photoionization, the abundances of reactive radicals are much larger than those of ions. However, many of the subsequent reactions will be destructive, with radicals reacting to break apart parent species they collide with. For example, $\mathrm{OH}$ can react with $\mathrm{NH}_{3}$ and $\mathrm{CH}_{4}$ to reform water and produce $\mathrm{NH}_{2}$ and $\mathrm{CH}_{3}$ respectively. A possible exception is the reaction of $\mathrm{CN}$ with hydrocarbons; these reactions typically result in the replacement of a $\mathrm{H}$ atom with the $-\mathrm{CN}$ group (Balucani et al., 2000). In particular, the reaction with acetylene $\left(\mathrm{C}_{2} \mathrm{H}_{2}\right)$ will lead to cyanoacetylene $\left(\mathrm{HC}_{3} \mathrm{~N}\right)$. Again, however, modeling shows that the resulting $\mathrm{HC}_{3} \mathrm{~N}$ yields are far less than observed in many comets (Rodgers and Charnley, 2001b). Neutral-neutral reactions do appear to be important in cometary sulfur chemistry. Based on our understanding of interstellar chemistry, S-bearing molecules take part in a predominately neutral chemistry. Canaves et al. $(2002,2004)$ have investigated the coma production of several S-bearing molecules, including NS and CS. They found very good agreement with the observed NS abundance in Comet HaleBopp and that the CS abundance should be approximately constant with cometocentric distance.

\subsection{Nonthermal Chemistry}

It has long been recognized that the energetic fragments produced in photodissociation reactions have the potential to drive a "suprathermal" chemistry in the coma (e.g., Huebner et al., 1991; Kissel et al., 1997). However, it was originally assumed that, in general, these reactions would be destructive. For example, $\mathrm{H}_{\mathrm{f}}$ atoms can react with water to form $\mathrm{H}_{2}$ and $\mathrm{OH}$. Hence the net effect of such reactions is simply to increase the quantum yield of $\mathrm{OH}$ atoms produced from photodestruction of water: In addition to the primary $\mathrm{OH}$ daughter, secondary $\mathrm{OH}$ radicals will also be produced.

More recent work has focused on the potential of suprathermal reactions to affect the coma chemistry in more interesting ways. Rodgers and Charnley $(1998,2001 \mathrm{c})$ looked at the possibility that $\mathrm{H}_{\mathrm{f}}$ atoms could isomerize $\mathrm{HCN}$ into its isomer $\mathrm{HNC}$, thus accounting for the puzzling $\mathrm{HNC} /$ $\mathrm{HCN}$ increase seen in several comets as they approach the Sun (e.g., Irvine et al., 1999; Biver et al., 2002; Rodgers et al., 2003). They showed that such reactions may be viable in large, active comets such as Hale-Bopp, but some other mechanism must account for the HNC production in smaller comets such as Hyakutake and Ikeya-Zhang (see Fig. 6). Pierce and A'Hearn (2003) examined the possibility that the reaction of $\mathrm{H}_{\mathrm{f}}$ atoms with $\mathrm{CO}_{2}$ could account for the extended source of $\mathrm{CO}$ seen in many comets. However, they conclude that this mechanism is an order of magnitude slower than direct photodissociation of $\mathrm{CO}_{2}$ into $\mathrm{CO}$. It is possible that the coma chemistry of sulfur could be strongly affected by $\mathrm{H}_{\mathrm{f}}$ atoms since these should readily abstract $\mathrm{H}$ 
atoms from the major parent, $\mathrm{H}_{2} \mathrm{~S}$. Similarly, more abundant coma molecules like $\mathrm{H}_{2} \mathrm{CO}$ and $\mathrm{CH}_{3} \mathrm{OH}$ could also be destroyed by $\mathrm{H}_{\mathrm{f}}$ atoms. The general applicability of these processes to coma composition remains to be investigated.

Other work has looked at the possibility of atoms produced in excited metastable states driving suprathermal reactions. For example, A'Hearn et al. (2000) suggested that $\mathrm{S}_{2}$ may be formed in the coma by the reaction of OCS with atomic sulfur produced in the singlet-D state. More recently, Glinski et al. (2003) showed that the reaction of $\mathrm{O}\left({ }^{3} \mathrm{P}\right)$ with $\mathrm{OH}$ may lead to significant $\mathrm{O}_{2}$ abundances in the inner coma. Another class of suprathermal reactions that are likely to be important are electron impact reactions. Although previous schemes have included these reactions (Boice et al., 1986), accurately calculating the rate at which they occur requires a detailed model of the hot electron energy distribution in the coma (e.g., Wegmann et al., 1999).

\subsection{Dust Fragmentation/Degradation and Extended Sources}

The inability of gas phase chemical reactions to generate sufficient quantities of particular molecules in the coma means that some other mechanism must be responsible for the extended sources of these molecules (Festou, 1999). Huebner (1987) proposed that destruction of POM in the coma could account for the formaldehyde source, and this was modeled by Boice et al. (1990). Based on their laboratory studies of POM degradation, Cottin et al. (2000) developed a more detailed model of thermal and photodegradation of POM in the coma. Matthews and Ludicky (1986) suggested that HCN polymers may be present in comets; degradation of these compounds may be an additional source of many small coma molecules (Rettig et al., 1992; Huebner and Boice, 1997), including HNC (Rodgers and Charnley, 2001c). Of course, in all these models there are a number of free parameters, and it is always possible to fit the observed abundances if one adjusts the initial abundance and/or the destruction rate of the mystery parent. Nevertheless, the models require abundances and destruction rates that are in agreement with rough theoretical estimates and laboratory measurements. Therefore, although these models do not prove that dust destruction is responsible for injection of $\mathrm{H}_{2} \mathrm{CO}$ and $\mathrm{HNC}$ into the coma, they certainly show that it is plausible.

\subsection{Isotopic Fractionation and Nuclear Spin Ratios}

Deuterium/hydrogen ratios have been observed in two coma species (HDO and $\mathrm{DCN}$ ), ${ }^{15} \mathrm{~N} /{ }^{14} \mathrm{~N}$ ratios in $\mathrm{CN}$ and $\mathrm{HCN}$, and ${ }^{13} \mathrm{C} /{ }^{12} \mathrm{C}$ ratios in $\mathrm{C}_{2}, \mathrm{CN}$, and $\mathrm{HCN}$; see Table 3 in Bockelée-Morvan et al. (2004) for a complete list. In the interstellar medium, proton transfer reactions are efficient in scrambling the D/H ratios among different species (e.g., Roberts and Millar, 2000). Hence, we might also expect some degree of mixing to occur in the coma. However, as discussed in section 5.2, ion-molecule chemistry cannot significantly alter the initial abundances of parent molecules sublimating from the nucleus, and detailed modeling shows that parent isotopic ratios are also unaffected (Rodgers and Charnley, 2002). An important consequence of this result is that measurements of isotope ratios can be used to test putative parent-daughter relationships. For example, although HCN undoubtedly accounts for some fraction of the $\mathrm{CN}$ observed in the coma, it has long been debated whether it can account for all the CN (Festou, 1999). Recent observations of $\mathrm{C}^{15} \mathrm{~N}$ in Comets LINEAR WM1 and Hale-Bopp by Arpigny et al. (2003) demonstrate that $\mathrm{CN}$ is significantly enhanced in ${ }^{15} \mathrm{~N}$, by around a factor of 2 , as compared with $\mathrm{HCN}$. Thus, HCN cannot be the sole parent of $\mathrm{CN}$, and if $\mathrm{HCN}$ contributes about half the $\mathrm{CN}$, the ${ }^{15} \mathrm{~N}$ ratio in the additional parent of $\mathrm{CN}$ must be even higher. If this parent is the same one that accounts for the extended source of HNC in comets, then one would also expect to see significant ${ }^{15} \mathrm{~N}$ enhancements in HNC. Similar observations of ${ }^{13} \mathrm{C}$-bearing isotopomers of $\mathrm{C}_{2}, \mathrm{C}_{3}$, and simple carbon-chain molecules (acetylene, ethene, ethane, cyanoacetylene) may also help to resolve the origin of these radicals.

As with molecular $\mathrm{D} / \mathrm{H}$ ratios, the nuclear spin ratios of cometary species reflect the temperatures at which they originated, and can be altered in the coma only marginally by proton transfer reactions. Ortho:para ratios (OPRs) have been observed in water in several comets (see BockeléeMorvan et al., 2004). Recently, Kawakita et al. (2002) have measured the OPR in $\mathrm{NH}_{2}$; since strict selection rules constrain the OPR in photodaughters as a function of the OPR in their parents, these observations can be used to probe the OPR in ammonia. Alternatively, one can turn this argument on its head and say that, if the OPR ratios in $\mathrm{NH}_{3}$ can be measured directly, the question of whether $\mathrm{NH}_{3}$ is the sole parent of $\mathrm{NH}_{2}$ can be answered. A similar investigation of the OPR in cometary formaldehyde may provide insights into the nature of its extended source.

\section{SUMMARY}

\subsection{Coma Physicochemistry}

In the inner regions of the coma, the outflowing gas behaves as a fluid, and fluid equations can be used to obtain a reasonable description of the flow. The gas initially cools as it expands adiabatically, but is eventually heated by thermalization of energetic photofragments. The ions are also heated by exoergic proton transfer reactions. The hydrodynamic approximation loses validity in the outer coma, where the density decreases, and many hot photoproducts are not thermalized. This is particularly important for electrons, and a very hot electron population exists in the outer coma. The chemistry and the physics are intimately coupled, and detailed models should include both. The chemistry is initiated by the solar radiation field, which produces reactive radicals and ions. Subsequent reactions in the coma, especially proton transfer reactions, result in the production 
of new coma species. However, although chemical reactions are extremely important in determining the relative abundances of different ions, they are unable to synthesize significant quantities of stable, neutral species. Therefore, unless an observed species is an obvious photodaughter, it is probable that it is present in the nuclear ice. Degradation of complex organic material is likely to contribute to the extended sources of some molecules, and studies of extended sources of molecules may provide insight into the nature of cometary CHON material. The energetic photodissociation products in the coma may drive a suprathermal chemistry. Studies of such processes are currently in their infancy.

\subsection{From Coma Measurements to Nucleus Properties}

Although almost all our information on comets comes from studies of the coma, what we really want to know is the nature of the cometary nucleus. As stressed in this chapter, detailed models of the coma are essential in order to calculate the density profiles and excitation states of coma molecules. However, even with these models, it is usually only possible to derive globally averaged properties in the extended coma. In terms of molecular production rates, this means that we can derive accurate values for the total gas release rates from the nucleus. However, when using these values to understand nucleus properties we encounter two important impediments. First, it is now known that gas is released not only from the nucleus surface, but also from subsurface layers (see Prialnik et al., 2004). Therefore, coma abundance ratios do not necessarily equal those in the icy nucleus, and models of the evolution of the cometary interior are required. Second, the nucleus is likely to be heterogeneous on small scales, and the gas production rate may vary dramatically with position (see Crifo et al., 2004). Hence, the total gas production rate will depend on the sum over the surface of many different regions, so it is not possible to simply derive "average" surface properties from the global gas production. Again, highly detailed modeling is essential to elucidate these issues.

\subsection{Open Questions and Future Directions}

The gas temperatures and velocities measured in situ in Comet Halley were in good agreement with coma models. It therefore appears that we have a relatively secure understanding of the physics in the coma. Chemically, the profiles and velocities of most parent molecules, as well as of many simple radicals and atoms, are in broad agreement with photodissociation models; nevertheless, many uncertainties remain. We briefly review several of the outstanding problems.

As discussed in section 3, the gas flow immediately above the nucleus surface is extremely complex. The gas is not in LTE, and interacts strongly with the entrained dust. Small-scale topography and heterogeneity of the nucleus can lead to steep spatial gradients in gas pressure and chemi- cal composition. Interaction of gas jets may result in standing shocks, and the divergence of chemically distinct jets may lead to chemical heterogeneity in the outer coma. A full understanding of the near-nucleus coma is vital if we are to use observations of the outer coma to infer the properties of the nucleus. These issues can only be resolved using extremely detailed three-dimensional gas-kinetic models; some results of the most up-to-date codes are presented in Crifo et al. (2004).

It appears that ion-molecule chemistry, of the kind that generates the diversity of molecules seen in the interstellar medium, is too slow to produce significant amounts of new species in the coma. However, the presence of highly energetic photodissociation products in the coma may allow a suprathermal chemistry to occur. Currently, only a small number of such reactions have been considered. Fast hydrogen atoms could play an important role here. For example, many sulfuretted molecules were detected for the first time in Comet Hale-Bopp, and it is known that the most important reactions in interstellar sulfur chemistry are neutral-neutral processes. Hydrogen abstraction from $\mathrm{H}_{2} \mathrm{~S}$ by $\mathrm{H}_{\mathrm{f}}$ could drive some reactions deep in the inner coma, generating new sulfuretted molecules. Clearly, there may exist many other possible reactions, not included in typical astrochemical networks, that can proceed efficiently in the coma. In particular, the global consequences of nonthermalized electrons in driving electron impact reactions has not been studied in sufficient depth.

The origin of the extended sources of certain molecules are not well known. Fragmentation of dust particles and/or macromolecules has been invoked to account for the additional sources of some molecules, such as $\mathrm{CO}$, formaldehyde, and several small radicals. Encouragingly, laboratory measurements of the properties of the proposed macromolecules appear to support this hypothesis (Cottin et al., 2000). More laboratory work on other possible $\mathrm{CHON}$ components is needed. Despite the fact that they have been seen in comets for decades, the origins of radicals such as $\mathrm{C}_{2}, \mathrm{C}_{3}$, and $\mathrm{CN}$ are still uncertain. However, measurements of isotopic ratios in these species and their putative parents may finally help to resolve this issue. Measurements of the OPR in formaldehyde may also help to constrain its source. Interferometric mapping of the coma of Hale-Bopp also appeared to reveal additional extended sources of molecules usually thought to be solely parent species, such as HCN and $\mathrm{CH}_{3} \mathrm{OH}$ (Blake et al., 1999; Kuan et al., 2003, 2004). The existence of such sources for other parent species would provide evidence that sublimation of volatiles from dust grains is occurring throughout the coma.

Given the rate of discovery (Bockelée-Morvan et al., 2004), it is likely that many more molecules will be discovered in future cometary apparitions, or through the reanalysis of archival data [e.g., the recent detection of ethylene glycol in Comet Hale-Bopp (Crovisier et al., 2004)]. Coma chemistry modeling will be necessary to identify the precise source of these molecules. Also, once the relevant laboratory data is available, future molecule discoveries will 
surely come from the existing large database of unidentified cometary lines (Feldman et al., 2004). It is also probable that many more isotopomers of the principal parent molecules will soon be detected. The resulting isotopic ratios will contain important clues about the origins of comets.

We have stressed in this chapter the coupling between the physics and chemistry in the coma. The chemistry and dynamics also strongly influence the excitation of coma molecules, particularly daughter molecules that will be produced in excited states, as well as polar molecules that can be excited by collisions with the hot electrons in the outer coma. Therefore, in order to fully understand the observed energy level distributions, combined dynamic-chemistryexcitation models are required. For example, Reylé and Boice (2003) developed such a model for the $\mathrm{S}_{2}$ excitation in the coma. Accurate calculations of the excitation, and its spatial variation, are particularly important when interpreting interferometric maps of the coma.

In the final analysis, coma chemistry models are only as good as the kinetic data they employ. As discussed in section 5.1, there are large uncertainties in some of the photodissociation and ionization rates used in these models. Rate coefficients for bimolecular reactions are typically taken from databases developed for modeling interstellar clouds [e.g., the UMIST ratefile (Le Teuff et al., 2000)]. Although many of the most important reactions in these schemes have been measured, there are many others for which experimental data is lacking. For the ion-molecule chemistry in the coma the most important reactions are exothermic proton transfer reactions; fortunately, many of these have been measured, or can safely be assumed to occur at the Langevin collisional rate. Regarding the possible suprathermal chemistry that may occur in the coma, however, very few reactions have been measured, and it is likely that there are many reactions currently omitted from interstellar reaction schemes that may be important in the coma. With the exception of POM, the product yields from photodegradation of proposed CHON constituents are also unknown. Finally, very few of the rates for collisional excitation have been measured; most values are simply estimates.

Acknowledgments. This work was supported by NASA's Planetary Atmospheres Program through NASA Ames Cooperative Agreement NCC2-1412 with the SETI Institute (S.D.R. and S.B.C.) and through NAG5-11052 to the Southwest Research Institute (W.F.H.), as well as by NSF Planetary Astronomy (9973186) (D.C.B.).

\section{REFERENCES}

A'Hearn M. F., Arpigny C., Feldman P. D., Jackson W. M., Meier R., Weaver H. A., Wellnitz D. D., and Woodney L. M. (2000) Formation of $\mathrm{S}_{2}$ in comets. Bull. Am. Astron. Soc., 32, 1079. Arpigny C., Jehin E., Manfroid J., Hutsemékers D., Schulz R., Stüwe J. A., Zucconi J-M., and Ilyin I. (2003) Anomalous nitrogen isotope ratio in comets. Science, 301, 1522-1525.

Balucani N., Asvany O., Huang L. C. L., Lee Y. T., Kaiser R. I., Osamura Y., and Bettinger H. F. (2000) Formation of nitriles in the interstellar medium via reactions of cyano radicals,
$\mathrm{CN}\left(\mathrm{X}^{2} \Sigma^{+}\right)$, with unsaturated hydrocarbons. Astrophys. J., 545, 892-906.

Bernstein M. P., Sandford S. A., Allamandola L. J., Chang S., and Scharberg M. A. (1995) Organic compounds produced by photolysis of realistic interstellar and cometary ice analogs containing methanol. Astrophys. J., 454, 327-344.

Biermann L. (1951) Kometenschweife und solare Korpuskularstrahlung. Z. Astrophys., 29, 274-286.

Biermann L., Giguere P. T., and Huebner W. F. (1982) A model of a comet coma with interstellar molecules in the nucleus. Astron. Astrophys., 108, 221-226.

Biver N. and 13 colleagues (1999) Spectroscopic monitoring of Comet C/1996 B2 (Hyakutake) with the JCMT and IRAM radio telescopes. Astron. J., 118, 1850-1872.

Biver N. and 22 colleagues (2002) The 1995-2002 long-term monitoring of Comet C/1995 O1 (Hale-Bopp) at radio wavelength. Earth Moon Planets, 90, 5-14.

Bockelée-Morvan D. and Crovisier J. (1987) The 2.7-micron water band of comet P/Halley - Interpretation of observations by an excitation model. Astron. Astrophys., 187, 425-430.

Bockelée-Morvan D., Crovisier J., and Gerard E. (1990) Retrieving the coma gas expansion velocity in $\mathrm{P} /$ Halley, Wilson (1987 VII) and several other comets from the 18-cm OH line shapes. Astron. Astrophys., 238, 382-400.

Bockelée-Morvan D. and 17 colleagues (2000) New molecules found in comet C/1995 O1 (Hale-Bopp). Investigating the link between cometary and interstellar material. Astron. Astrophys., 353, 1101-1114.

Bockelée-Morvan D., Crovisier J., Mumma M. J., and Weaver H. A. (2004) The composition of cometary volatiles. In Comets II (M. C. Festou et al., eds.), this volume. Univ. of Arizona, Tucson.

Boice D. C., Huebner W. F., Keady J. J., Schmidt H. U., and Wegmann R. (1986) A model of Comet P/Giacobini-Zinner. Geophys. Res. Lett., 13, 381-384.

Boice D. C., Huebner W. F., Sablik M. J., and Konno I. (1990) Distributed coma sources and the $\mathrm{CH}_{4} / \mathrm{CO}$ ratio in comet Halley. Geophys. Res. Lett., 17, 1813-1816.

Blake G. A., Qi C., Hogerheijde M. R., Gurwell M. A., and Muhleman D. O. (1999) Sublimation from icy jets as a probe of the interstellar volatile content of comets. Nature, 398, 213216.

Canaves M. V., de Almeida A. A., Boice D. C., and Sanzovo G. C. (2002) Nitrogen sulfide in Comets Hyakutake (C/1996 B2) and Hale-Bopp (C/1995 O1). Earth Moon Planets, 90, 335-347.

Canaves M. V., de Almeida A. A., Boice D. C., and Sanzovo G. C. (2004) Chemistry of NS and CS in cometary comae. Adv. Space Res., in press.

Combi M. R. and Smyth W. H. (1988) Monte Carlo particle-trajectory models for neutral cometary gases. I - Models and equations. Astrophys. J., 327, 1026-1059.

Combi M. R., Harris W. M., and Smyth W. H. (2004) Gas dynamics and kinetics in the cometary coma: Theory and observations. In Comets II (M. C. Festou et al., eds.), this volume. Univ. of Arizona, Tucson.

Cottin H., Gazeau M. C., Doussin J. F., and Raulin F. (2000) An experimental study of the photodegradation of polyoxymethylene at 122, 147 and 193 nm. J. Photochem. Photobiology A, 135, 53-64.

Cottin H., Gazeau M. C., Benilan Y., and Raulin F. (2001) Polyoxymethylene as parent molecule for the formaldehyde extended source in Comet Halley. Astrophys. J., 556, 417-420. 
Cravens T. E. (1991) Plasma processes in the inner coma. In Comets in the Post-Halley Era (R. L. Newburn Jr. et al., eds.), pp. 1211-1255. Kluwer, Dordrecht.

Cravens T. E. and Körösmezey A. (1986) Vibrational and rotational cooling of electrons by water vapor. Planet. Space Sci., 34, 961-970.

Crifo J. (1991) Hydrodynamic models of the collisional coma. In Comets in the Post-Halley Era (R. L. Newburn Jr. et al., eds.), pp. 937-989. Kluwer, Dordrecht.

Crifo J. F., Fulle M., Kömle N. I., and Szegö K. (2004) Nucleuscoma structural relationships: Lessons from physical models. In Comets II (M. C. Festou et al., eds.), this volume. Univ. of Arizona, Tucson.

Crovisier J. (1984) The water molecule in comets - fluorescence mechanisms and thermodynamics of the inner coma. Astron. Astrophys., 130, 361-372.

Crovisier J. (1989) The photodissociation of water in cometary atmospheres. Astron. Astrophys., 213, 459-464.

Crovisier J. (2004) The molecular complexity of comets. In Astrobiology: Future Perspectives (P. Ehrenfreund et al., eds.), in press. Kluwer, Dordrecht.

Crovisier J., Bockelée-Morvan D., Biver N., Colom P., Despois D., and Lis D. C. (2004) Ethylene glycol in comet C/1995 O1 (Hale-Bopp). Astron. Astrophys., in press.

Draine B. T. (1980) Interstellar shock waves with magnetic precursors. Astrophys. J., 241, 1021-1038.

Draine B. T. (1986) Multicomponent, reacting MHD flows. Mon. Not. R. Astron. Soc., 220, 133-148.

Eberhardt P. and Krankowsky D. (1995) The electron temperature in the inner coma of comet P/Halley. Astron. Astrophys. 295, 795-806.

Eberhardt P. and 10 colleagues (1987) The $\mathrm{CO}$ and $\mathrm{N}_{2}$ abundance in comet P/Halley. Astron. Astrophys., 187, 481-484.

Feldman P. D., Cochran A. L., and Combi M. R. (2004) Spectroscopic investigations of fragment species in the coma. In Comets II (M. C. Festou et al., eds.), this volume. Univ. of Arizona, Tucson.

Festou M. C. (1981) The density distribution of neutral compounds in cometary atmospheres. I - Models and equations. Astron. Astrophys., 95, 69-79.

Festou M. C. (1999) On the existence of distributed sources in comet comae. Space Sci. Rev., 90, 53-67.

Festou M. C., Keller H. U., Bertaux J. L., and Barker E. S. (1983) Lyman-alpha observations of comets West 1976 VI and P d'Arrest 1976 XI with Copernicus. Astrophys. J., 265, 925 932.

Friedlander S. K. (1977) Smoke, Dust and Haze. Wiley, New York. $317 \mathrm{pp}$.

Gail H. P. and Sedlmayr E. (1985) Dust formation in stellar winds - II. Carbon condensation in stationary, spherically expanding winds. Astron. Astrophys., 148, 183-190.

Geiss J., Altwegg K., Anders E., Balsiger H., Meier A., Shelley E. G., Ip W.-H., Rosenbauer H., and Neugebauer M. (1991) Interpretation of the ion mass spectra in the mass per charge range 25-35 amu/e obtained in the inner coma of Halley's comet by the HIS-sensor of the Giotto IMS experiment. Astron. Astrophys., 247, 226-234.

Giguere P. T. and Huebner W. F. (1978) A model of comet comae. I. Gas-phase chemistry in one dimension. Astrophys. J., 223, 638-654.

Glinski R. J., Harris W. M., Anderson C. M., and Morgenthaler J. P. (2003) Oxygen/hydrogen chemistry in inner comae of active comets. In XXVth General Assembly: Highlights of Astronomy, Vol. 13 (O. Engvole, ed.), in press. Astronomical Society of the Pacific, San Francisco.

Gombosi T. I., Cravens T. E., and Nagy A. F. (1985) Time-dependent dusty gas dynamical flow near cometary nuclei. Astrophys. J., 293, 328-341.

Gombosi T. I., Nagy A. F., and Cravens T. E. (1986) Dust and neutral gas modeling of the inner atmospheres of comets. Rev. Geophys., 24, 667-700.

Harich S. A., Yang X. F., Yang X., and Dixon R. N. (2001) Extremely rotationally excited $\mathrm{OH}$ from water (HOD) photodissociation through conical intersections. Phys. Rev. Lett., 87, 253201.

Harker D. E., Wooden D. H., Woodward C. E., and Lisse C. M. (2002) Grain properties of Comet C/1995 O1 (Hale-Bopp). Astrophys. J., 580, 579-597.

Haser L. (1957) Distribution d'intensite dans la tete d'une comete. Bull. Acad. R. Sci. Liege, 43, 740-750.

Hodges R. R. (1990) Monte Carlo simulation of nonadiabatic expansion in cometary atmospheres - Halley. Icarus, 83, 410433.

Huebner W. F. (1985) Cometary comae. In Molecular Astrophysics: State of the Art and Future Directions (G. H. F. Diercksen et al., eds.), pp. 311-330. Reidel, Dordrecht.

Huebner W. F. (1987) First polymer in space identified in Comet Halley. Science, 237, 628-630.

Huebner W. F. and Benkhoff J. (1999) From coma abundances to nucleus composition. Space Sci. Rev., 90, 117-130.

Huebner W. F. and Boice D. C. (1997) Polymers and other macromolecules in comets. In Comets and the Origin and Evolution of Life (P. J. Thomas et al., eds.), pp. 111-129. Springer-Verlag, New York.

Huebner W. F. and Giguere P. T. (1980) A model of comet comae. II. Effects of solar photodissociative ionization. Astrophys. J., 238, 753-762.

Huebner W. F. and Keady J. J. (1984) First-flight escape from spheres with $\mathrm{R}^{-2}$ density distribution. Astron. Astrophys., 135, 177-180.

Huebner W. F., Boice D. C., Schmidt H. U., and Wegmann R. (1991) Structure of the coma: Chemistry and solar wind interaction. In Comets in the Post-Halley Era (R. L. Newburn Jr. et al., eds.), pp. 907-936. Kluwer, Dordrecht.

Huebner W. F., Keady J. J., and Lyon S. P. (1992) Solar photo rates for planetary atmospheres and atmospheric pollutants. Astrophys. Space Sci., 195, 1-294.

Ip W-H. (1983) On photochemical heating of cometary comae The cases of $\mathrm{H}_{2} \mathrm{O}$ and CO-rich comets. Astrophys. J., 264, 726-732.

Ip W-H. (2004) Global solar wind interaction and ionospheric dynamics. In Comets II (M. C. Festou et al., eds.), this volume. Univ. of Arizona, Tucson.

Irvine W. M., Dickens J. E., Lovell A. J., Schloerb F. P., Senay M., Bergin E. A., Jewitt D., and Matthews H. E. (1998) Chemistry in cometary comae. Faraday Discuss., 109, 475-492.

Irvine W. M., Dickens J. E., Lovell A. J., Schloerb F. P., Senay M., Bergin E. A., Jewitt D., and Matthews H. E. (1999) The HNC/HCN ratio in comets. Earth Moon Planets, 78, 29-35.

Jackson W. M., Xu D., Huang J., Price R. J., and Volman D. H. (2002) New experimental and theoretical techniques for studying photochemical reactions of cometary atmospheres. Earth Moon Planets, 89, 197-220.

Kawakita H., Watanabi J., Fuse T., Furusho R., and Abe S. (2002) 
Spin temperature of ammonia determined from $\mathrm{NH}_{2}$ in Comet C/2001 A2 (LINEAR). Earth Moon Planets, 90, 371-379.

Kissel J. and 18 colleagues (1986) Composition of comet Halley dust particles from Giotto observations. Nature, 321, 336-337.

Kissel J., Jrueger F. R., and Roessler K. (1997) Organic chemistry in comets from remote and in situ observations. In Comets and the Origin and Evolution of Life (P. J. Thomas et al., eds.), pp. 69-109. Springer-Verlag, New York.

Kitamura Y. (1986) Axisymmetric dusty gas jet in the inner coma of a comet. Icarus, 66, 241-257.

Konno I., Huebner W. F., and Boice D. C. (1993) A model of dust fragmentation in near-nucleus jet-like features on Comet $\mathrm{P} /$ Halley. Icarus, 101, 84-94.

Körösmezey A. and Gombosi T. I. (1990) A time-dependent dusty gas dynamic model of axisymmetric cometary jets. Icarus, 84 , 118-153.

Körösmezey A., Cravens T. E., Nagy A. F., Gombosi T. I., and Mendis D. A. (1987) A new model of cometary ionospheres. $J$. Geophys. Res., 92, 7331-7340.

Krankowsky D. and 11 colleagues (1986) In situ gas and ion measurements at comet Halley. Nature, 321, 326-329.

Kuan Y.-J., Huang H.-C., Snyder L. E., Veal J. M., Woodney L. M., Forster J. R., Wright M. C. H., and A'Hearn M. F. (2003) BIMA array observations of cometary organic molecules in Hale-Bopp. In Proceedings of the XIIth Rencontres de Blois on Frontiers of Life (L. M. Celnikier and J. Tran Thanh Van, eds.), pp. 55-57. The Gioi, Vietnam.

Kuan Y.-J., Charnley S.B., Huang H.-C., Kisiel Z., Ehrenfreund P., Tseng W.-L., and Yan C.-H. (2004) Searches for interstellar molecules of potential prebiotic importance. Adv. Space Res., 33, 31-39.

Le Teuff Y. H., Millar T. J., and Markwick A. J. (2000) The UMIST database for astrochemistry 1999. Astron. Astrophys. Suppl. Ser., 146, 157-168.

Lisse C. M., Cravens T. E., and Dennerl K. (2004) X-ray and extreme ultraviolet emission from comets. In Comets II (M. C. Festou et al., eds.), this volume. Univ. of Arizona, Tucson.

Lovell A. J., Schloerb F. P., Bergin E. A., Dickens J. E., De Vries C. H., Senay M. C., and Irvine W. M. (1999) $\mathrm{HCO}^{+}$in the coma of Comet Hale-Bopp. Earth Moon Planets, 77, 253-258.

Marconi M. L. and Mendis D. A. (1982) The photochemical heating of the cometary atmosphere. Astrophys. J., 260, 386-394.

Marconi M. L. and Mendis D. A. (1983) The atmosphere of a dirty-clathrate cometary nucleus - A two-phase, multifluid model. Astrophys. J., 273, 381-396.

Marconi M. L. and Mendis D. A. (1986) The electron density and temperature in the tail of comet Giacobini-Zinner. Geophys. Res. Lett., 13, 405-406.

Matthews C. N. and Ludicky R. (1986) The dark nucleus of comet Halley: Hydrogen cyanide polymers. In 20th ESLAB Symposium of Halley's Comet (B. Battrick et al., eds.), pp. 273-277. ESA SP-250, Noordwijk, The Netherlands.

McCoy R. P., Meier R. R., Keller H. U., Opal C. B., and Carruthers G. R. (1992) The hydrogen coma of Comet P/Halley observed in Lyman-alpha using sounding rockets. Astron. Astrophys., 258, 555-565.

Meier R., Eberhardt P., Krankowsky D., and Hodges R. R. (1993) The extended formaldehyde source in comet P/Halley. Astron. Astrophys., 277, 677-690.

Mitchell G. F., Prasad S. S., and Huntress W. T. (1981) Chemical model calculations of $\mathrm{C}_{2}, \mathrm{C}_{3}, \mathrm{CH}, \mathrm{CN}, \mathrm{OH}$, and $\mathrm{NH}_{2}$ abundances in cometary comae. Astrophys. J., 244, 1087-1093.
Neubauer F. M. (1991) The magnetic field structure of the cometary plasma environment. In Comets in the Post-Halley Era (R. L. Newburn Jr. et al., eds.), pp. 1107-1124. Kluwer, Dordrecht.

Neubauer F. M. and 11 colleagues (1986) First results from the Giotto magnetometer experiment at comet Halley. Nature, 321, 352-355.

Nordholt J. E. and 14 colleagues (2003) Deep Space 1 encounter with Comet 19P/Borrelly: Ion composition measurements by the PEPE mass spectrometer. Geophys. Res. Lett., 30, 14651468.

Oppenheimer M. (1975) Gas phase chemistry in comets. Astrophys. J., 196, 251-259.

Pack J. L., Voshall R. E., and Phelps A. V. (1962) Drift velocities of slow electrons in krypton, xenon, deuterium, carbon monoxide, carbon dioxide, water vapor, nitrous oxide, and ammonia. Phys. Rev., 127, 2084-2089.

Pierce D. M. and A'Hearn M. F. (2003) Formation of carbon monoxide in the near-nucleus coma of comets. Bull. Am. Astron. Soc., 35, 968.

Prialnik D., Benkhoff J., and Podolak M. (2004) Modeling the structure and activity of comet nuclei. In Comets II (M. C. Festou et al., eds.), this volume. Univ. of Arizona, Tucson.

Putnam A. (1961) Integrable form of droplet drag coefficient. ARS J., 31, 1467-1468.

Rettig T. W., Tegler S. C., Pasto D. J., and Mumma M. J. (1992) Comet outbursts and polymers of HCN. Astrophys. J., 398, 293-298.

Reylé C. and Boice D. C. (2003) An $\mathrm{S}_{2}$ fluorescence model for interpreting high-resolution cometary spectra. I. Model description and initial results. Astrophys. J., 587, 464-471.

Roberts H. and Millar T. J. (2000) Modelling of deuterium chemistry and its application to molecular clouds. Astron. Astrophys., 361, 388-398.

Rodgers S. D. and Charnley S. B. (1998) HNC and HCN in comets. Astrophys. J. Lett., 501, L227-L230.

Rodgers S. D. and Charnley S. B. (2001a) Chemical differentiation in regions of massive star formation. Astrophys. J., 546, 324-329.

Rodgers S. D. and Charnley S. B. (2001b) On the origin of HNC in Comet Lee. Mon. Not. R. Astron. Soc., 323, 84-92.

Rodgers S. D. and Charnley S. B. (2001c) Organic synthesis in the coma of Comet Hale-Bopp? Mon. Not. R. Astron. Soc., 320, L61-L64.

Rodgers S. D. and Charnley S. B. (2002) A model of the chemistry in cometary comae: Deuterated molecules. Mon. Not. R. Astron. Soc., 330, 660-674.

Rodgers S. D., Butner H. M., Charnley S. B., and Ehrenfreund P. (2003) The HNC/HCN ratio in comets: Observations of $\mathrm{C} /$ 2002 C1 (Ikeya-Zhang). Adv. Space Res., 31, 2577-2582.

Schleicher D. G. and Farnham T. L. (2004) Photometry and imaging of the coma with narrowband filters. In Comets II (M. C. Festou et al., eds.), this volume. Univ. of Arizona, Tucson.

Schmidt H. U., Wegmann R., Huebner W. F., and Boice D. C. (1988) Cometary gas and plasma flow with detailed chemistry. Comp. Phys. Comm., 49, 17-59.

Shimizu M. (1976) The structure of cometary atmospheres. I Temperature distribution. Astrophys. Space Sci., 40, 149-155.

Su T. and Bowers M. T. (1973) Theory of ion-polar molecule collisions. J. Chem. Phys., 58, 3027-3037.

Swings P. (1943) Cometary spectra. Mon. Not. R. Astron. Soc., 103, 86-111. 
Wegmann R., Schmidt H. U., Huebner W. F., and Boice D. C. (1987) Cometary MHD and chemistry. Astron. Astrophys., 187, 339-350.

Wegmann R., Jockers K., and Bonev T. (1999) $\mathrm{H}_{2} \mathrm{O}^{+}$ions in comets: Models and observations. Planet. Space Sci., 47, 745-763.

Whipple F. L. (1950) A comet model. Part I. The acceleration of Comet Encke. Astrophys. J., 111, 375-394.
Whipple F. L. (1951) A comet model. Part II. Physical relations for comets and meteors. Astrophys. J., 113, 464-474.

Whipple F. L. and Huebner W. F. (1976) Physical processes in comets. Annu. Rev. Astron. Astrophys., 14, 143-172.

Xie X. and Mumma M. J. (1992) The effect of electron collisions on rotational populations of cometary water. Astrophys. J., 386, 720-728. 